\title{
An investigation of polyhedral deformation in two mixed-metal diarsenates: $\mathrm{SrZnAs}_{2} \mathrm{O}_{7}$ and $\mathrm{BaCuAs}_{2} \mathrm{O}_{7}$
}

\section{Sabina Kovač, Ljiljana Karanović and Tamara Đorđević}

Acta Cryst. (2015). C71, 330-337

\section{H IUCr Journals CRYSTALLOGRAPHY JOURNALS ONLINE \\ Copyright (C) International Union of Crystallography \\ Author(s) of this paper may load this reprint on their own web site or institutional repository provided that this cover page is retained. Republication of this article or its storage in electronic databases other than as specified above is not permitted without prior permission in writing from the IUCr. \\ For further information see http://journals.iucr.org/services/authorrights.html}




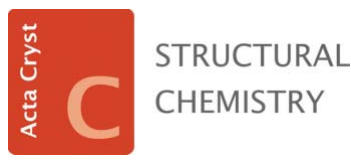

ISSN 2053-2296

Received 21 January 2015

Accepted 18 March 2015

Edited by A. L. Spek, Utrecht University, The Netherlands

Keywords: diarsenates; strontium-zinc; barium-copper; hydrothermal synthesis; X-ray diffraction; crystal structure; polyhedral deformation.

CCDC references: 1054886; 1054885

Supporting information: this article has supporting information at journals.iucr.org/c

\section{An investigation of polyhedral deformation in two mixed-metal diarsenates: $\mathrm{SrZnAs}_{2} \mathrm{O}_{7}$ and $\mathrm{BaCuAs}_{2} \mathrm{O}_{7}$}

\author{
Sabina Kovač, ${ }^{a *}$ Ljiljana Karanović ${ }^{a}$ and Tamara Dorđevićb
}

a University of Belgrade, Faculty of Mining and Geology, Laboratory of Crystallography, Đušina 7, 11000 Belgrade, Serbia, and ${ }^{\mathbf{b}}$ Universität Wien, Institut für Mineralogie und Kristallographie, Althansstrasse 14, A-1090 Vienna, Austria. *Correspondence e-mail: sabinakovac@yahoo.com

Two isostructural diarsenates, $\mathrm{SrZnAs}_{2} \mathrm{O}_{7}$ (strontium zinc diarsenate), (I), and $\mathrm{BaCuAs}_{2} \mathrm{O}_{7}$ [barium copper(II) diarsenate], (II), have been synthesized under hydrothermal conditions and characterized by single-crystal X-ray diffraction. The three-dimensional open-framework crystal structure consists of cornersharing $\mathrm{M2O}_{5}(\mathrm{M} 2=\mathrm{Zn}$ or $\mathrm{Cu})$ square pyramids and diarsenate $\left(\mathrm{As}_{2} \mathrm{O}_{7}\right)$ groups. Each $\mathrm{As}_{2} \mathrm{O}_{7}$ group shares its five corners with five different $M 2 \mathrm{O}_{5}$ square pyramids. The resulting framework delimits two types of tunnels aligned parallel to the [010] and [100] directions where the large divalent nine-coordinated $M 1$ $(M 1=\mathrm{Sr}$ or $\mathrm{Ba})$ cations are located. The geometrical characteristics of the $M 1 \mathrm{O}_{9}, M 2 \mathrm{O}_{5}$ and $\mathrm{As}_{2} \mathrm{O}_{7}$ groups of known isostructural diarsenates, adopting the general formula $M 1^{\mathrm{II}} M 2^{\mathrm{II}} \mathrm{As}_{2} \mathrm{O}_{7}\left(M 1^{\mathrm{II}}=\mathrm{Sr}, \mathrm{Ba}, \mathrm{Pb} ; M 2^{\mathrm{II}}=\mathrm{Mg}, \mathrm{Co}, \mathrm{Cu}, \mathrm{Zn}\right)$ and crystallizing in the space group $P 2_{1} / n$, are presented and discussed.

\section{Introduction}

The structural properties of divalent cation diphosphates and diarsenates, $M_{2}^{\mathrm{II}} X_{2}^{\mathrm{V}} \mathrm{O}_{7}\left(M^{\mathrm{II}}=\right.$ divalent cation; $X^{\mathrm{V}}=\mathrm{P}$, As) (Weil et al., 2009; Edhokkar et al., 2012), mixed-valence cation diphosphates and diarsenates, $M 1^{\mathrm{I}} M 2^{\mathrm{III}} X^{\mathrm{V}}{ }_{2} \mathrm{O}_{7}$ (Kolitsch, 2004; Schwendtner \& Kolitsch, 2004; Horchani-Naifer \& Férid, 2007; Ouerfelli et al., 2007; Rousse et al., 2013) and $M 1_{2}^{\mathrm{I}} M 2^{\mathrm{II}} X_{2}^{\mathrm{V}} \mathrm{O}_{7}$ (Faggiani \& Calvo, 1976; Marzouki et al., 2012), and mixed-cation diphosphates with the general formula $M 1^{\mathrm{II}} M 2^{\mathrm{II}} \mathrm{P}_{2} \mathrm{O}_{7}\left(M 1^{\mathrm{II}}=\mathrm{Ca}, \mathrm{Sr}, \mathrm{Ba}, \mathrm{Pb} ; M 2^{\mathrm{II}}=\mathrm{Mg}, \mathrm{Cr}, \mathrm{Mn}, \mathrm{Fe}\right.$, $\mathrm{Co}, \mathrm{Ni}, \mathrm{Cu}, \mathrm{Zn}, \mathrm{Cd}$; references are given in the footnotes of Table 1) have been broadly investigated and described. Yet, reports on the $M 1^{\mathrm{II}} M 2^{\mathrm{II}} \mathrm{As}_{2} \mathrm{O}_{7}$ diarsenates are rather rare. Prior to this study, the structural data for only seven diarsenates have been reported, namely $\mathrm{SrCuAs}_{2} \mathrm{O}_{7}$ (Chen \& Wang, 1996), $\mathrm{SrCoAs}_{2} \mathrm{O}_{7}$ (Horng \& Wang, 1994), $\mathrm{PbCuAs}_{2} \mathrm{O}_{7}$ (Pertlik, 1986), $\mathrm{BaCuAs}_{2} \mathrm{O}_{7}$ (Wardojo \& Hwu, 1995; Chen \& Wang, 1996), $\mathrm{BaMgAs}_{2} \mathrm{O}_{7}$ and $\mathrm{BaCoAs}_{2} \mathrm{O}_{7}$ (Mihajlović et al., 2004), and $\mathrm{BaZnAs}_{2} \mathrm{O}_{7}$ (Đorđević, 2008).

All known diphosphate and diarsenate $M 1^{\mathrm{II}} M 2^{\mathrm{II}} X_{2} \mathrm{O}_{7}$ compounds, crystallizing in the monoclinic space group $P 2_{1} / n$ with comparable pseudo-orthorhombic unit-cell dimensions $a \simeq 5.2-5.7, b \simeq 8.2-8.9, c \simeq 12.5-13.3 \AA$ and $\beta \simeq 90^{\circ}$, show a structural similarity to the $\alpha-\mathrm{Ca}_{2} \mathrm{P}_{2} \mathrm{O}_{7}$ structure (Calvo, 1968). The structure of $\alpha-\mathrm{Ca}_{2} \mathrm{P}_{2} \mathrm{O}_{7}$ is characterized by two crystallographically distinct eight-coordinated cationic sites occupied by $\mathrm{Ca} 1$ and $\mathrm{Ca} 2$. In the $\alpha-\mathrm{Ca}_{2} \mathrm{P}_{2} \mathrm{O}_{7}$ isotype compounds $M 1^{\mathrm{II}} M 2^{\mathrm{II}} X_{2} \mathrm{O}_{7}$, the $M 1^{\mathrm{II}}$ cation of larger radius occupies the Ca1 site, whereas the smaller $M 2^{\mathrm{II}}$ cation occupies the $\mathrm{Ca} 2$ site. The coordination numbers of $M 1^{\mathrm{II}}$ and $M 2^{\mathrm{II}}$ may vary 
Table 1

Comparison of the unit-cell parameters $\left(\AA{ }^{\circ}\right)$ for $M 1^{\mathrm{II}} M 2^{\mathrm{II}} X_{2} \mathrm{O}_{7}$ compounds $\left(M 1=\mathrm{Ba}^{2+}, \mathrm{Sr}^{2+}, \mathrm{Pb}^{2+}, \mathrm{Ca}^{2+} ; \mathrm{M2}^{2}=\mathrm{Cu}^{2+}, \mathrm{Mg}^{2+}, \mathrm{Zn}^{2+}, \mathrm{Ni}^{2+}, \mathrm{Co}^{2+}, \mathrm{Cr}^{2+} ; X=\right.$ $\left.\mathrm{As}^{5+}, \mathrm{P}^{5+}\right)$.

\begin{tabular}{|c|c|c|c|c|c|c|}
\hline Compound & $a$ & $b$ & $c$ & $\beta$ & $V\left(\AA^{3}\right)$ & $\begin{array}{l}\text { ICSD (2014) } \\
\text { reference }\end{array}$ \\
\hline $\mathrm{SrCuAs}_{2} \mathrm{O}_{7}{ }^{\mathbf{1}}$ & $5.550(1)$ & 8.299 (2) & $12.858(2)$ & 91.75 (3) & $592.0(2)$ & 82624 \\
\hline $\mathrm{SrCoAs}_{2} \mathrm{O}_{7}^{2}$ & $5.520(1)$ & $8.391(2)$ & $13.083(3)$ & $90.90(2)$ & $605.9(2)$ & 74545 \\
\hline $\mathrm{SrZnAs}_{2} \mathrm{O}_{7}{ }^{\mathbf{3}}$ & $5.5114(11)$ & $8.3524(17)$ & $13.106(3)$ & $91.01(3)$ & $603.2(2)$ & \\
\hline $\mathrm{PbCuAs}_{2} \mathrm{O}_{7}{ }^{4}$ & $5.553(1)$ & $8.404(1)$ & $13.011(2)$ & $91.61(2)$ & $606.95(16)$ & 61339 \\
\hline $\mathrm{BaCuAs}_{2} \mathrm{O}_{7}^{1}$ & $5.736(1)$ & $8.458(2)$ & $13.044(3)$ & $91.16(3)$ & $632.7(2)$ & 82625 \\
\hline $\mathrm{BaCuAs}_{2} \mathrm{O}_{7}^{\mathbf{3}}$ & $5.7343(11)$ & $8.4722(17)$ & $13.071(3)$ & $91.12(3)$ & $634.9(2)$ & \\
\hline $\mathrm{BaCuAs}_{2} \mathrm{O}_{7}^{\mathbf{5}}$ & $5.740(5)$ & $8.475(3)$ & $13.090(3)$ & $91.24(4)$ & $636.6(6)$ & 80329 \\
\hline $\mathrm{BaMgAs}_{2} \mathrm{O}_{7}{ }^{6}$ & $5.620(1)$ & $8.629(2)$ & $13.344(3)$ & $90.20(3)$ & $647.1(2)$ & \\
\hline $\mathrm{BaCoAs}_{2} \mathrm{O}_{7}^{6}$ & $5.649(1)$ & 8.577 (1) & $13.278(2)$ & 90.014 (1) & $643.3(2)$ & \\
\hline $\mathrm{BaZnAs}_{2} \mathrm{O}_{7}{ }^{7}$ & $5.6260(10)$ & $8.557(2)$ & $13.317(3)$ & $90.01(3)$ & $641.1(2)$ & 260061 \\
\hline $\mathrm{CaCuP}_{2} \mathrm{O}_{7}^{\mathbf{8}^{\prime}}$ & $5.2104(4)$ & $8.0574(5)$ & $12.344(1)$ & $91.356(6)$ & $518.08(7)$ & 68510 \\
\hline$\alpha-\mathrm{Ca}_{2} \mathrm{P}_{2} \mathrm{O}_{7}{ }^{9}$ & $5.315(5)$ & $8.542(8)$ & $12.66(1)$ & $90.3(1)$ & $574.8(9)$ & 22225 \\
\hline $\mathrm{SrNiP}_{2} \mathrm{O}_{7}^{\mathbf{1 0}}$ & $5.2691(5)$ & $8.2674(8)$ & $12.6140(13)$ & $90.246(9)$ & $549.48(9)$ & 411740 \\
\hline $\mathrm{SrCuP}_{2} \mathrm{O}_{7}{ }^{\mathbf{1 1}}$ & $5.369(1)$ & 8.129 (1) & $12.455(2)$ & 90.59 (1) & $543.56(15)$ & 74445 \\
\hline $\mathrm{SrMgP}_{2} \mathrm{O}_{7}^{\mathbf{1 2}}$ & $5.3046(8)$ & $8.3053(13)$ & $12.700(2)$ & $90.502(3)$ & $559.48(15)$ & 280782 \\
\hline $\mathrm{SrCoP}_{2} \mathrm{O}_{7}{ }^{13}$ & $5.3165(4)$ & $8.2574(5)$ & $12.6755(7)$ & $90.133(5)$ & $556.46(6)$ & 69578 \\
\hline $\mathrm{SrZnP}_{2} \mathrm{O}_{7}{ }^{\mathbf{1 4}}$ & $5.3143(2)$ & $8.2080(3)$ & $12.7250(6)$ & 90.192 (4) & $555.06(4)$ & \\
\hline $\mathrm{SrZnP}_{2} \mathrm{O}_{7}{ }^{15}$ & $5.30906(2)$ & $8.21392(3)$ & $12.73595(5)$ & $90.1573(3)$ & $555.390(5)$ & 160198 \\
\hline $\mathrm{SrFeP}_{2} \mathrm{O}_{7}^{\mathbf{1 6}}$ & $5.370(2)$ & $8.268(4)$ & $12.693(3)$ & $90.37(2)$ & $563.5(4)$ & 280078 \\
\hline $\mathrm{SrCrP}_{2} \mathrm{O}_{7}^{17}$ & $5.422(2)$ & $8.3254(19)$ & $12.542(4)$ & $90.39(3)$ & $566.1(3)$ & 280309 \\
\hline $\mathrm{SrCdP}_{2} \mathrm{O}_{7}{ }^{\mathbf{1 8}}$ & $5.414(1)$ & $8.615(3)$ & $12.878(5)$ & $90.01(3)$ & $600.6(4)$ & 72672 \\
\hline $\mathrm{Sr}_{1.34} \mathrm{~Pb}_{0.66} \mathrm{P}_{2} \mathrm{O}_{7}{ }^{19}$ & $5.433(4)$ & $8.944(6)$ & $13.183(6)$ & $90.12(5)$ & $640.6(7)$ & 75000 \\
\hline $\mathrm{PbCuP}_{2} \mathrm{O}_{7}^{20}$ & $5.381(1)$ & $8.194(2)$ & $12.569(1)$ & $90.39(1)$ & $554.18(18)$ & 79996 \\
\hline $\mathrm{PbCoP}_{2} \mathrm{O}_{7}{ }^{\mathbf{2 0}}$ & $5.322(1)$ & $8.292(2)$ & $12.777(2)$ & $90.18(1)$ & $563.85(19)$ & 79994 \\
\hline $\mathrm{PbMnP}_{2} \mathrm{O}_{7}{ }^{\mathbf{2 0}}$ & $5.393(1)$ & $8.461(2)$ & $12.848(3)$ & $90.00(3)$ & $586.3(2)$ & 79995 \\
\hline $\mathrm{BaMgP}_{2} \mathrm{O}_{7}^{\mathbf{2 1}}$ & $5.483(1)$ & $8.561(3)$ & $12.626(2)$ & $91.32(2)$ & $592.5(3)$ & 39398 \\
\hline $\mathrm{BaMnP}_{2} \mathrm{O}_{7}{ }^{22}$ & $5.5537(6)$ & $8.633(2)$ & $12.825(2)$ & 90.08 (1) & $614.90(18)$ & 78657 \\
\hline
\end{tabular}

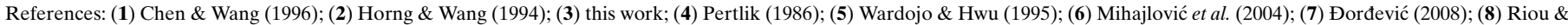

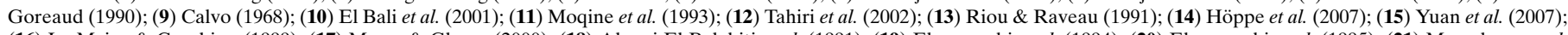

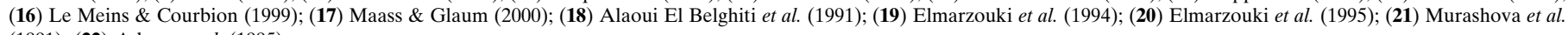
(1991); (22) Adams et al. (1995).

from 7 to 9 for $M 1^{\mathrm{II}}$ and from 5 to 9 for $M 2^{\mathrm{II}}$. The $X^{\mathrm{V}}$ is the smallest tetrahedrally coordinated cation. The $X_{2} \mathrm{O}_{7}{ }^{4-}$ anion consists of two $\mathrm{XO}_{4}$ tetrahedra sharing an $\mathrm{O}$ atom, denoted $\mathrm{O}_{\mathrm{b}}$, with a bridging bond angle $X-\mathrm{O}_{\mathrm{b}}-X$ of approximately $125^{\circ}$. Therefore, the structure of $M 1^{\mathrm{II}} M 2^{\mathrm{II}} X_{2} \mathrm{O}_{7}$ compounds can be described as ionic with $M 1^{\mathrm{II}}$ and $M 2^{\mathrm{II}}$ as the cations and $X_{2} \mathrm{O}_{7}{ }^{4-}$ as the anion. Alternatively, the structure can be described as the complex compound $\left\{M 1\left[M 2\left(X_{2} \mathrm{O}_{7}\right)\right]\right\}_{n}$, with $M 2$ as a central metal cation surrounded by five $X_{2} \mathrm{O}_{7}$ ligands. $M 1$ serves as a counter-cation in order to produce electroneutrality. The structures of diarsenates $M 1^{\mathrm{II}} M 2^{\mathrm{II}} \mathrm{As}_{2} \mathrm{O}_{7}$ are characterized by a three-dimensional open framework formed from corner-sharing $M 2 \mathrm{O}_{5}$ square pyramids and $\mathrm{As}_{2} \mathrm{O}_{7}$ groups. The framework contains two types of tunnels, viz. smaller pentagonal tunnels along the [100] direction and larger tunnels along the [010] direction, in which arrays of $M 1^{\mathrm{II}}$ cations are located.

The present article reports the hydrothermal synthesis and crystal structures of $\mathrm{SrZnAs}_{2} \mathrm{O}_{7}$ and $\mathrm{BaCuAs}_{2} \mathrm{O}_{7}$ based on single-crystal X-ray diffraction data. $\mathrm{SrZnAs}_{2} \mathrm{O}_{7}$ is a compound with a new chemical composition and the structure of $\mathrm{BaCuAs}_{2} \mathrm{O}_{7}$ has been redetermined. The lattice parameters obtained for $\mathrm{BaCuAs}_{2} \mathrm{O}_{7}$ have lower s.u. values and deviate somewhat from the values reported earlier by Wardojo \& Hwu (1995) and Chen \& Wang (1996). In $\mathrm{SrZnAs}_{2} \mathrm{O}_{7}$ and $\mathrm{BaCuAs}_{2} \mathrm{O}_{7}$, the alkaline earth $\mathrm{Sr}$ and $\mathrm{Ba}$ atoms occupy the $M 1^{\mathrm{II}}$ site [coordination number $(\mathrm{CN})=9$ ], whereas $\mathrm{Zn}$ and $\mathrm{Cu}$ atoms occupy the $M 2^{\mathrm{II}}$ site $(\mathrm{CN}=5)$. To investigate how cations of different type, size and coordination characteristics are accommodated and how $\mathrm{As}_{2} \mathrm{O}_{7}$ groups are adopted, we have studied the distortion of the coordination polyhedra and the conformation of the $\mathrm{As}_{2} \mathrm{O}_{7}$ group.

\section{Experimental}

\subsection{Synthesis}

Single crystals of both $\mathrm{SrZnAs}_{2} \mathrm{O}_{7}$ and $\mathrm{BaCuAs}_{2} \mathrm{O}_{7}$ were obtained as reaction products from the following mixtures (using an approximate 1:1:1 molar ratio in both cases) and distilled water. $\mathrm{SrZnAs}_{2} \mathrm{O}_{7}$ was obtained from a mixture of $\mathrm{ZnO}$ (Sigma-Aldrich, 99.99\%), $\mathrm{Sr}(\mathrm{OH})_{2} \cdot 8 \mathrm{H}_{2} \mathrm{O}$ (SigmaAldrich, 95\%) and $\mathrm{As}_{2} \mathrm{O}_{5}$ (Alfa Products, $>99.9 \%$ ), and $\mathrm{BaCuAs}_{2} \mathrm{O}_{7}$ was obtained from a mixture of $\mathrm{Ba}(\mathrm{OH})_{2} \cdot 8 \mathrm{H}_{2} \mathrm{O}$ (Fluka, >97\%), $\mathrm{Cu}\left(\mathrm{NO}_{3}\right)_{2} \cdot \mathrm{H}_{2} \mathrm{O}$ (Sigma-Aldrich, >98\%) and $\mathrm{As}_{2} \mathrm{O}_{3}$ (Alfa Aesar, $>99.9 \%$ ). The initial $\mathrm{pH}$ of both mixtures was 3. The Teflon vessels were enclosed in stainless steel autoclaves and both mixtures were heated under autogenous pressure. The first mixture was heated from room temperature to $493 \mathrm{~K}$ over a period of $4 \mathrm{~h}$, held at that temperature for $72 \mathrm{~h}$ and cooled slowly to room temperature over a period of $72 \mathrm{~h}$. The second mixture was heated from room temperature to $473 \mathrm{~K}$ over a period of $4 \mathrm{~h}$, held at that temperature for $52 \mathrm{~h}$ and cooled slowly to room temperature over a period of $152 \mathrm{~h}$. 
Table 2

Experimental details.

$\mathrm{SrZnAs}_{2} \mathrm{O}_{7}$

$\mathrm{SrZnAs}_{2} \mathrm{O}_{7}$
414.85
Monoclinic, $P 2_{1} / n$
298
$5.5114(11), 8.3524$ (17), $13.106(3)$
$91.01(3)$
$603.2(2)$
4
$\mathrm{Mo} \mathrm{K \alpha}$
23.69
$0.16 \times 0.03 \times 0.02$

Nonius KappaCCD diffractometer Multi-scan (Otwinowski \& Minor, 1997) $0.668,1.000$

$7055,1835,1555$

$T_{\min }, T_{\max }$

No. of measured, independent and observed $[I>2 \sigma(I)]$ reflections

$R_{\text {int }}$

$(\sin \theta / \lambda)_{\max }\left(\AA^{-1}\right)$

0.062

0.714

Refinement

$R\left[F^{2}>2 \sigma\left(F^{2}\right)\right], w R\left(F^{2}\right), S$

No. of reflections

No. of parameters

$\Delta \rho_{\max }, \Delta \rho_{\min }\left(\mathrm{e} \AA^{-3}\right)$

Computer programs: COLLECT (Nonius, 2002), SCALEPACK (Otwinowski \& Minor, 1997), DENZO-SMN (Otwinowski et al., 2003), SIR97 (Altomare et al., 1999), SHELXL2013 (Sheldrick, 2015), WinGX (Farrugia, 2012), PLATON (Spek, 2009), ATOMS (Dowty, 2000) and publCIF (Westrip, 2010).

At the end of the reaction, the $\mathrm{pH}$ of the first solvent was not measured because the sample was dry, while the $\mathrm{pH}$ of the second solvent was again 3 . All reaction products were filtered off, washed thoroughly with distilled water and dried in air. $\mathrm{SrZnAs}_{2} \mathrm{O}_{7}$ crystallized as colourless elongated prismatic crystals (yield $\mathrm{ca} 45 \%$ ) up to $0.18 \mathrm{~mm}$ in length, together with prisms of $\mathrm{Sr}\left(\mathrm{AsO}_{3} \mathrm{OH}\right)$ (Mihajlović \& Effenberger, 2006) (yield $\mathrm{ca} 40 \%$ ) and undissolved $\mathrm{ZnO}$ powder. $\mathrm{BaCuAs}_{2} \mathrm{O}_{7}$ crystallized as prismatic blue-green crystals (yield ca 50\%) up to $0.08 \mathrm{~mm}$ in length, together with a new $\mathrm{Ba}-\mathrm{Cu}$ arsenate (yield 50\%) (results to be published elsewhere).

\subsection{Refinement}

Crystal data, data collection and structure refinement details are summarized in Table 2. Selected bond distances and angles are listed in Tables 3 and 4 for $\mathrm{SrZnAs}_{2} \mathrm{O}_{7}$ and $\mathrm{BaCuAs}_{2} \mathrm{O}_{7}$, respectively.

\section{Results and discussion}

The unit-cell parameters of known isostructural diphosphates and diarsenates with the general formula $M 1^{\mathrm{II}} M 2^{\mathrm{II}} X_{2} \mathrm{O}_{7}$ $\left(M 1^{\mathrm{II}}=\mathrm{Ca}, \mathrm{Sr}, \mathrm{Ba}, \mathrm{Pb} ; M 2^{\mathrm{II}}=\mathrm{Mg}, \mathrm{Cr}, \mathrm{Mn}, \mathrm{Fe}, \mathrm{Co}, \mathrm{Ni}, \mathrm{Cu}, \mathrm{Zn}\right.$, $\mathrm{Cd} ; X^{\mathrm{V}}=\mathrm{P}, \mathrm{As}$ ) are given in Table 1. Generally, the increase of the unit-cell volume directly follows the increase of the ionic radii of the divalent $M 1$ and $M 2$ cations. The unit-cell volumes for diarsenates are in the range 592.0 (2)-647.1 (2) $\AA^{3}$, while they are smaller than $592 \AA^{3}$ for diphosphates, except for
$\mathrm{BaCuAs}_{2} \mathrm{O}_{7}$

\author{
$\mathrm{BaCuAs}_{2} \mathrm{O}_{7}$ \\ 462.72 \\ Monoclinic, $P 2_{1} / n$ \\ 298 \\ 5.7343 (11), 8.4722 (17), 13.071 (3) \\ $91.12(3)$ \\ $634.9(2)$ \\ Mo $K \alpha$ \\ 19.84 \\ $0.06 \times 0.03 \times 0.02$
}

Nonius KappaCCD diffractometer

Multi-scan (Otwinowski \& Minor, 1997)

$0.807,1.000$

4991, 1298, 1232

0.025

0.625

$0.025,0.059,1.20$

1297

101

$2.28,-0.72$
$\mathrm{SrCdP}_{2} \mathrm{O}_{7}, \mathrm{Sr}_{1.34} \mathrm{~Pb}_{0.66} \mathrm{P}_{2} \mathrm{O}_{7}, \mathrm{BaMgP}_{2} \mathrm{O}_{7}$ and $\mathrm{BaMnP}_{2} \mathrm{O}_{7}$. It should be noted that the $M 2^{\mathrm{II}}$ ionic radii $(0.65-0.68 \AA)$ for diarsenates listed in Table 1 have very similar values, while the $M 1^{\mathrm{II}}$ ionic radii for $\mathrm{CN}=9$ are $1.31,1.35$ and $1.47 \AA$ for $\mathrm{Sr}, \mathrm{Pb}$ and $\mathrm{Ba}$, respectively. This indicates that the unit-cell volumes for known $M 1^{\mathrm{II}} M 2^{\mathrm{II}} \mathrm{As}_{2} \mathrm{O}_{7}$ compounds depend mainly on the size of the $M 1^{\mathrm{II}}$ ionic radii. Earlier studies (Maass \& Glaum, 2000) have shown that the unit-cell parameters of the series of diphosphates $\mathrm{Sr} M 2 \mathrm{P}_{2} \mathrm{O}_{7}(M 2=\mathrm{Cr}, \mathrm{Mn}, \mathrm{Fe}, \mathrm{Co}, \mathrm{Ni}, \mathrm{Cu}, \mathrm{Zn}, \mathrm{Cd})$ are dependent on the size of the $M 2$ cation too. It was also found (Brown \& Calvo, 1970; Alaoui El Belghiti et al., 1991; Chen \& Wang, 1996; Yuan et al., 2007) that there is no strong correlation between the $M 1$ and $M 2$ cation sizes and the structure type of the $M 1^{\mathrm{II}} M 2^{\mathrm{II}} \mathrm{As}_{2} \mathrm{O}_{7}$ diarsenates, as was found in the corresponding diphosphates. This is because the $\mathrm{As}_{2} \mathrm{O}_{7}$ group is adjustable to the bonding requirements of other groups in the structure by tetrahedral distortion, by changing the As $-\mathrm{O}_{\mathrm{b}}-\mathrm{As}$ angle as well as the dihedral angles $\mathrm{O}_{\mathrm{t}}-$ As1 $\cdots$ As2 $-\mathrm{O}_{\mathrm{t}}$ between the two $\mathrm{AsO}_{4}$ tetrahedra (Chen \& Wang, 1996).

The tetrahedral distortion can be expressed numerically via the quadratic elongation, $\lambda$, and the tetrahedral angle variances, $\sigma_{\text {tet }}^{2}$ (Robinson et al., 1971); the values were calculated using the VOLCAL program (Hazen \& Finger, 1982) as implemented in Win $G X$ (Farrugia, 2012). Since the bridging $\left(\mathrm{O}_{\mathrm{b}}\right)$ and six terminal $\left(\mathrm{O}_{\mathrm{t}}\right) \mathrm{O}$ atoms of the $\mathrm{As}_{2} \mathrm{O}_{7}$ group of $M 1^{\mathrm{II}} \mathrm{M}^{\mathrm{II}} \mathrm{As}_{2} \mathrm{O}_{7}$ are located in general positions, three symmetrically independent dihedral or torsion angles can be distinguished within the group. Similar to tetrahedral angle 
Table 3

Selected geometric parameters $\left(\AA,^{\circ}\right)$ for $\mathrm{SrZnAs}_{2} \mathrm{O}_{7}$.

\begin{tabular}{|c|c|c|c|}
\hline $\mathrm{Sr} 1-\mathrm{O} 2$ & $2.525(3)$ & $\mathrm{As} 1-\mathrm{O} 1^{\mathrm{iii}}$ & $1.671(3)$ \\
\hline $\mathrm{Sr} 1-\mathrm{O} 1^{\mathrm{i}}$ & $2.557(3)$ & As1-O4 & $1.744(3)$ \\
\hline $\mathrm{Sr} 1-\mathrm{O} 2^{\mathrm{ii}}$ & $2.576(3)$ & As $2-O 5^{\mathrm{v}}$ & $1.669(3)$ \\
\hline $\mathrm{Sr} 1-\mathrm{O} 6^{\mathrm{iii}}$ & $2.602(3)$ & As $2-O 7^{\text {iv }}$ & $1.679(3)$ \\
\hline $\mathrm{Sr} 1-\mathrm{O} 3^{\mathrm{ii}}$ & $2.640(3)$ & As2-O6 & $1.682(3)$ \\
\hline $\mathrm{Sr} 1-\mathrm{O} 7^{\mathrm{iv}}$ & $2.714(3)$ & As $2-O 4^{\mathrm{ii}}$ & $1.749(3)$ \\
\hline $\mathrm{Sr} 1-\mathrm{O} 5^{\mathrm{i}}$ & $2.765(3)$ & $\mathrm{Zn} 1-\mathrm{O} 3$ & $1.973(3)$ \\
\hline $\mathrm{Sr} 1-\mathrm{O} 1^{\mathrm{iii}}$ & $2.851(3)$ & $\mathrm{Zn} 1-\mathrm{O} 5$ & $2.013(3)$ \\
\hline $\mathrm{Sr} 1-\mathrm{O} 5^{\mathrm{iii}}$ & $3.268(3)$ & $\mathrm{Zn} 1-\mathrm{O} 6$ & $2.023(3)$ \\
\hline $\mathrm{As} 1-\mathrm{O} 2^{\mathrm{iii}}$ & $1.654(3)$ & $\mathrm{Zn} 1-\mathrm{O} 7$ & $2.106(3)$ \\
\hline $\mathrm{As} 1-\mathrm{O} 3^{\mathrm{ii}}$ & $1.670(3)$ & $\mathrm{Zn} 1-\mathrm{O} 1$ & 2.157 (3) \\
\hline $\mathrm{O} 2-\mathrm{Sr} 1-\mathrm{O} 1^{\mathrm{i}}$ & $93.44(10)$ & $\mathrm{O} 2^{\mathrm{ii}}-\mathrm{Sr} 1-\mathrm{O} 5^{\mathrm{iii}}$ & $139.81(9)$ \\
\hline $\mathrm{O} 2-\mathrm{Sr} 1-\mathrm{O} 2^{\mathrm{ii}}$ & $155.50(7)$ & $\mathrm{O} 6^{\mathrm{iii}}-\mathrm{Sr} 1-\mathrm{O} 5^{\mathrm{iii}}$ & $61.41(9)$ \\
\hline $\mathrm{O} 1^{\mathrm{i}}-\mathrm{Sr} 1-\mathrm{O} 2^{\mathrm{ii}}$ & $69.30(10)$ & $\mathrm{O} 3^{\mathrm{ii}}-\mathrm{Sr} 1-\mathrm{O} 5^{\mathrm{iii}}$ & $106.87(9)$ \\
\hline $\mathrm{O} 2-\mathrm{Sr} 1-\mathrm{O} 6^{\mathrm{iii}}$ & $120.91(10)$ & $\mathrm{O} 7^{\mathrm{iv}}-\mathrm{Sr} 1-\mathrm{O}^{5 \mathrm{iii}}$ & $60.98(9)$ \\
\hline $\mathrm{O} 1^{\mathrm{i}}-\mathrm{Sr} 1-\mathrm{O}^{\mathrm{iii}}$ & $140.59(10)$ & $\mathrm{O} 5^{\mathrm{i}}-\mathrm{Sr} 1-\mathrm{O} 5^{\mathrm{iii}}$ & $146.28(9)$ \\
\hline $\mathrm{O} 2^{\mathrm{ii}}-\mathrm{Sr} 1-\mathrm{O} 6^{\mathrm{iii}}$ & $81.88(10)$ & $\mathrm{O} 1^{\mathrm{iii}}-\mathrm{Sr} 1-\mathrm{O} 5^{\mathrm{iii}}$ & $50.28(8)$ \\
\hline $\mathrm{O} 2-\mathrm{Sr} 1-\mathrm{O} 3^{\mathrm{ii}}$ & $99.39(10)$ & $\mathrm{O} 2^{\mathrm{iii}}-\mathrm{As} 1-\mathrm{O} 3^{\mathrm{ii}}$ & $114.79(16)$ \\
\hline $\mathrm{O} 1^{\mathrm{i}}-\mathrm{Sr} 1-\mathrm{O} 3^{\mathrm{ii}}$ & $120.53(10)$ & $\mathrm{O} 2^{\mathrm{iii}}-\mathrm{As} 1-\mathrm{O} 1^{\mathrm{iii}}$ & $113.26(16)$ \\
\hline $\mathrm{O} 2^{\mathrm{ii}}-\mathrm{Sr} 1-\mathrm{O} 3^{\mathrm{ii}}$ & $76.47(10)$ & $\mathrm{O} 3^{\mathrm{ii}}-\mathrm{As} 1-\mathrm{O} 1^{\mathrm{iii}}$ & $106.36(16)$ \\
\hline $\mathrm{O} 6^{\mathrm{iii}}-\mathrm{Sr} 1-\mathrm{O} 3^{\mathrm{ii}}$ & $75.40(10)$ & $\mathrm{O} 2^{\mathrm{iii}}-\mathrm{As} 1-\mathrm{O} 4$ & $108.51(17)$ \\
\hline $\mathrm{O} 2-\mathrm{Sr} 1-\mathrm{O} 7^{\text {iv }}$ & $98.52(11)$ & $\mathrm{O} 3^{\mathrm{ii}}-\mathrm{As} 1-\mathrm{O} 4$ & $105.51(16)$ \\
\hline $\mathrm{O} 1^{\mathrm{i}}-\mathrm{Sr} 1-\mathrm{O}^{\mathrm{iv}}$ & $81.41(10)$ & $\mathrm{O} 1^{\mathrm{iii}}-\mathrm{As} 1-\mathrm{O} 4$ & 107.95 \\
\hline $\mathrm{O} 2^{\mathrm{ii}}-\mathrm{Sr} 1-\mathrm{O} 7^{\mathrm{iv}}$ & $95.96(10)$ & $\mathrm{O} 5^{\mathrm{v}}-\mathrm{As} 2-\mathrm{O}^{\mathrm{iv}}$ & $118.74(15)$ \\
\hline $\mathrm{O} 6^{\mathrm{iii}}-\mathrm{Sr} 1-\mathrm{O} 7^{\mathrm{iv}}$ & $75.32(9)$ & $\mathrm{O} 5^{\mathrm{v}}-\mathrm{As} 2-\mathrm{O} 6$ & $111.75(16)$ \\
\hline $\mathrm{O} 3^{\mathrm{ii}}-\mathrm{Sr} 1-\mathrm{O}^{\mathrm{iv}}$ & $150.51(10)$ & $\mathrm{O} 7^{\mathrm{iv}}-\mathrm{As} 2-\mathrm{O} 6$ & $109.62(15)$ \\
\hline $\mathrm{O} 2-\mathrm{Sr} 1-\mathrm{O} 5^{\mathrm{i}}$ & $83.80(10)$ & $\mathrm{O} 5^{\mathrm{v}}-\mathrm{As} 2-\mathrm{O} 4^{\mathrm{ii}}$ & $101.56(15)$ \\
\hline $\mathrm{O} 1^{\mathrm{i}}-\mathrm{Sr} 1-\mathrm{O} 5^{\mathrm{i}}$ & $59.01(10)$ & $\mathrm{O} 7^{\mathrm{iv}}-\mathrm{As} 2-\mathrm{O} 4^{\mathrm{ii}}$ & 108.69 \\
\hline $\mathrm{O} 2^{\mathrm{ii}}-\mathrm{Sr} 1-\mathrm{O} 5^{\mathrm{i}}$ & $72.49(10)$ & $\mathrm{O} 6-\mathrm{As} 2-\mathrm{O} 4^{\mathrm{ii}}$ & $105.33(15)$ \\
\hline $\mathrm{O} 6^{\mathrm{iii}}-\mathrm{Sr} 1-\mathrm{O}^{\mathrm{i}}$ & $136.65(10)$ & $\mathrm{O} 3-\mathrm{Zn} 1-\mathrm{O} 5$ & 116.04 (13) \\
\hline $\mathrm{O} 3^{\mathrm{ii}}-\mathrm{Sr} 1-\mathrm{O} 5^{\mathrm{i}}$ & $65.08(9)$ & $\mathrm{O} 3-\mathrm{Zn} 1-\mathrm{O} 6$ & 142.82 \\
\hline $\mathrm{O} 7^{\mathrm{iv}}-\mathrm{Sr} 1-\mathrm{O} 5^{\mathrm{i}}$ & $140.40(9)$ & $\mathrm{O} 5-\mathrm{Zn} 1-\mathrm{O} 6$ & $98.26(13)$ \\
\hline $\mathrm{O} 2-\mathrm{Sr} 1-\mathrm{O} 1^{\mathrm{iii}}$ & $65.43(9)$ & $\mathrm{O} 3-\mathrm{Zn} 1-\mathrm{O} 7$ & $85.04(13)$ \\
\hline $\mathrm{O} 1^{\mathrm{i}}-\mathrm{Sr} 1-\mathrm{O} 1^{\mathrm{iii}}$ & $156.84(6)$ & $\mathrm{O} 5-\mathrm{Zn} 1-\mathrm{O} 7$ & $118.29(12)$ \\
\hline $\mathrm{O} 2^{\mathrm{ii}}-\mathrm{Sr} 1-\mathrm{O} 1^{\mathrm{iii}}$ & $127.02(9)$ & $\mathrm{O} 6-\mathrm{Zn} 1-\mathrm{O} 7$ & $91.26(12)$ \\
\hline $\mathrm{O} 6^{\mathrm{iii}}-\mathrm{Sr} 1-\mathrm{O} 1^{\mathrm{iii}}$ & $62.52(9)$ & $\mathrm{O} 3-\mathrm{Zn} 1-\mathrm{O} 1$ & $87.94(13)$ \\
\hline $\mathrm{O}^{\mathrm{ii}}-\mathrm{Sr} 1-\mathrm{O} 1^{\mathrm{iii}}$ & $58.16(9)$ & $\mathrm{O} 5-\mathrm{Zn} 1-\mathrm{O} 1$ & $78.02(12)$ \\
\hline $\mathrm{O} 7^{\mathrm{iv}}-\mathrm{Sr} 1-\mathrm{O} 1^{\mathrm{iii}}$ & $109.88(9)$ & $\mathrm{O} 6-\mathrm{Zn} 1-\mathrm{O} 1$ & $85.46(12)$ \\
\hline $\mathrm{O} 5^{\mathrm{i}}-\mathrm{Sr} 1-\mathrm{O} 1^{\mathrm{iii}}$ & $106.95(9)$ & $\mathrm{O} 7-\mathrm{Zn} 1-\mathrm{O} 1$ & $163.69(12)$ \\
\hline $\mathrm{O} 2-\mathrm{Sr} 1-\mathrm{O} 5^{\mathrm{iii}}$ & $64.66(10)$ & $\mathrm{As} 1-\mathrm{O} 4-\mathrm{As} 2^{\mathrm{vi}}$ & $122.39(18)$ \\
\hline $\mathrm{O} 1^{\mathrm{i}}-\mathrm{Sr} 1-\mathrm{O} 5^{\mathrm{iii}}$ & $130.69(9)$ & & \\
\hline
\end{tabular}

Symmetry codes: (i) $-x+\frac{3}{2}, y+\frac{1}{2},-z+\frac{1}{2}$; (ii) $-x+\frac{1}{2}, y+\frac{1}{2},-z+\frac{1}{2}$; (iii) $x-1, y, z$; (iv) $-x+1,-y+1,-z ;(\mathrm{v})-x+2,-y+1,-z ;$ (vi) $-x+\frac{1}{2}, y-\frac{1}{2},-z+\frac{1}{2}$.

variances, $\sigma_{\text {tet }}^{2}\left[=\Sigma\left(\theta_{i}-109.47\right)^{2} / 5, i=1,2,3,4,5,6\right]$, the formula $\sigma_{\text {dih }}^{2}=\Sigma\left(\theta_{i}-60\right)^{2} / 3(i=1,2,3)$ was used to calculate the dihedral angle variances and the dihedral angles were calculated using the program PLATON (Spek, 2009). Diarsenate groups in $M 1^{\mathrm{II}} M 2^{\mathrm{II}} \mathrm{As}_{2} \mathrm{O}_{7}$ compounds involve two crystallographically non-equivalent $\mathrm{AsO}_{4}$ tetrahedra (Fig. 1). In the structures of $\mathrm{SrZnAs}_{2} \mathrm{O}_{7}$ and $\mathrm{BaCuAs}_{2} \mathrm{O}_{7}$, nearly identical average $\langle A s 1-\mathrm{O}\rangle$ bond lengths were found (Table 5). The mean $\langle$ As2 $-\mathrm{O}\rangle$ distances in $\mathrm{SrZnAs}_{2} \mathrm{O}_{7}$ and $\mathrm{BaCuAs}_{2} \mathrm{O}_{7}$ are also almost identical and longer than the corresponding values for $\langle\mathrm{As} 1-\mathrm{O}\rangle$, which correlate with the distinct angular distortions in the $\mathrm{As}_{10} \mathrm{O}_{4}\left(\sigma_{\text {tet }}^{2}=14.24\right.$ and 14.96) and $\mathrm{As} 2 \mathrm{O}_{4}$ $\left(\sigma_{\text {tet }}^{2}=34.26\right.$ and 34.80) tetrahedra (Table 5 and Fig. 2). The longest As $-\mathrm{O}$ bonds are to the bridging $\mathrm{O} 4$ atom. The As1O4-As2 bond angles are $122.39(18)$ and $125.3(2)^{\circ}$ in $\mathrm{SrZnAs}_{2} \mathrm{O}_{7}$ and $\mathrm{BaCuAs}_{2} \mathrm{O}_{7}$, respectively, which compare well with the same bridging angles of $122.7(3)^{\circ}$ in $\mathrm{SrCoAs}_{2} \mathrm{O}_{7}$ (Horng \& Wang, 1994), $122.7(3)^{\circ}$ in $\mathrm{SrCuAs}_{2} \mathrm{O}_{7}$ (Chen \& Wang, 1996), as well as values of 125.9 (3) (Wardojo \& Hwu, 1995) and $124.7(6)^{\circ}$ (Chen \& Wang, 1996) in two different forms of $\mathrm{BaCuAs}_{2} \mathrm{O}_{7}$. In $\mathrm{SrZnAs}_{2} \mathrm{O}_{7}$ and $\mathrm{BaCuAs}_{2} \mathrm{O}_{7}$, two $\mathrm{AsO}_{4}$ tetrahedra of the diarsenate group form a staggered conformation [dihedral angles O1-As1‥As2-O7, O2-

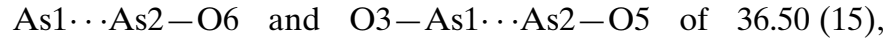
$40.93(16)$ and $69.86(19)^{\circ}$, respectively, for $\mathrm{SrZnAs}_{2} \mathrm{O}_{7}$, and 42.7 (2), 48.1 (2) and $74.0(3)^{\circ}$, respectively, for $\mathrm{BaCuAs}_{2} \mathrm{O}_{7}$ ]. The dihedral angle variances, $\sigma_{\mathrm{dih}}^{2}$, are 337.71 and 212.30 for $\mathrm{SrZnAs}_{2} \mathrm{O}_{7}$ and $\mathrm{BaCuAs}_{2} \mathrm{O}_{7}$, respectively. This points to a greater $\mathrm{As}_{2} \mathrm{O}_{7}$ angular deviation from the ideal value of $60^{\circ}$ in $\mathrm{SrZnAs}_{2} \mathrm{O}_{7}$ than in $\mathrm{BaCuAs}_{2} \mathrm{O}_{7}$ (Table 5 and Fig. 2).

Each diarsenate group shares its five corners with five different $M 2 \mathrm{O}_{5}$ square pyramids. This leaves one corner free $\left(\mathrm{O} 2\right.$ in $\mathrm{SrZnAs}_{2} \mathrm{O}_{7}$ and $\left.\mathrm{BaCuAs}_{2} \mathrm{O}_{7}\right)$ and this participates in the coordination of the $M 1^{\mathrm{II}}$ cation. The $M 2$ position is characterized by a square-pyramidal geometry and a $4+1$ bond

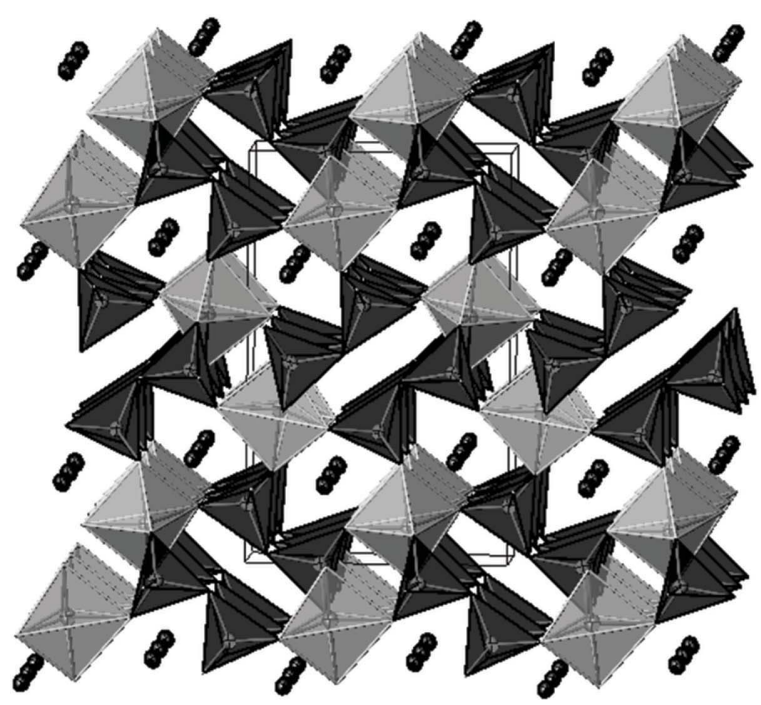

(a)

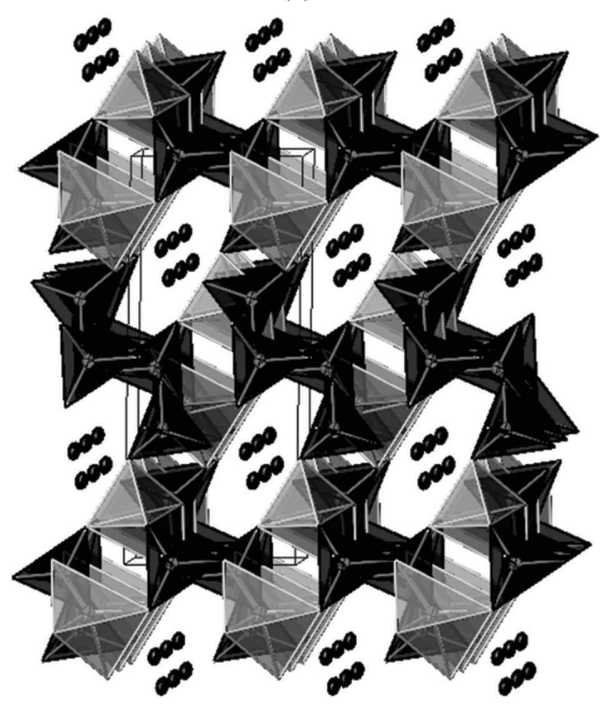

(b)

Figure 1

Polyhedral views of the framework in the $\mathrm{SrZnAs}_{2} \mathrm{O}_{7}$ structure $(a)$ along the [100] direction (the $c$ axis is vertical) and $(b)$ along the [010] direction (the $c$ axis is vertical). A trace of the unit cell is drawn for reference. $\mathrm{AsO}_{4}$ tetrahedra are dark grey, $\mathrm{ZnO}_{5}$ pyramids are light grey and $\mathrm{Sr}$ atoms are represented by black spheres. 
distance scheme. The average lengths of the four basal $M 2-\mathrm{O}$ bonds are 2.029 (3) and 1.977 (4) $\AA$, and the fifth axial bond is longer, i.e. 2.157 (3) and 2.382 (4) $\AA$, for $\mathrm{SrZnAs}_{2} \mathrm{O}_{7}$ and $\mathrm{BaCuAs}_{2} \mathrm{O}_{7}$, respectively. The mean $\langle\mathrm{Zn}-\mathrm{O}\rangle$ and $\langle\mathrm{Cu}-\mathrm{O}\rangle$ distances are 2.054 (3) and 2.060 (4) $\AA$. They can be compared to the $\langle M 2-\mathrm{O}\rangle$ distances of $2.062 \AA$ found in $\mathrm{BaZnAs}_{2} \mathrm{O}_{7}$ (Đorđević, 2008) and 2.035 $\AA$ found in $\mathrm{SrCuAs}_{2} \mathrm{O}_{7}$ (Chen \& Wang, 1996). In both structures, the $M 2$ atom deviates from the least-squares basal plane [0.4683 (5) and 0.2411 (7) $\AA$ for $\mathrm{Zn}$ and $\mathrm{Cu}$, respectively] towards the axial $\mathrm{O}$ atom. As reported previously (Riou \& Raveau, 1991; Horng \& Wang, 1994; Wardojo \& Hwu, 1995), the M2 atoms tend to be located close to the centre of the $M 2 \mathrm{O}_{5}$ pyramid, unlike $\mathrm{Cu}$ atoms, which are located closer to the square basal plane, leading to a long $\mathrm{Cu}-\mathrm{O}$ apical distance. This is in agreement with the Jahn-Teller effect of the $\mathrm{Cu}^{\mathrm{II}}$ cation.

Considering the bond distances in $\mathrm{SrZnAs}_{2} \mathrm{O}_{7}$ and $\mathrm{BaCuAs}_{2} \mathrm{O}_{7}$, the coordination of the $\mathrm{Sr} / \mathrm{Ba}$-hosting site $M 1$ with $\mathrm{CN}=9$ is approximately $3+3+3$ and can be described as a distorted tricapped trigonal prism. The average $\langle\mathrm{Sr}-\mathrm{O}\rangle$ and $\langle\mathrm{Ba}-\mathrm{O}\rangle$ bond distances are 2.722 (3) and 2.821 (4) $\AA$, which compare well with the average $\langle\mathrm{Sr}-\mathrm{O}\rangle$ and $\langle\mathrm{Ba}-\mathrm{O}\rangle$ bond distances of $2.705 \AA$ in $\mathrm{SrCuAs}_{2} \mathrm{O}_{7}$ (Chen \& Wang, 1996) and $2.829 \AA$ in $\mathrm{BaCoAs}_{2} \mathrm{O}_{7}$ (Mihajlović et al., 2004). It should be
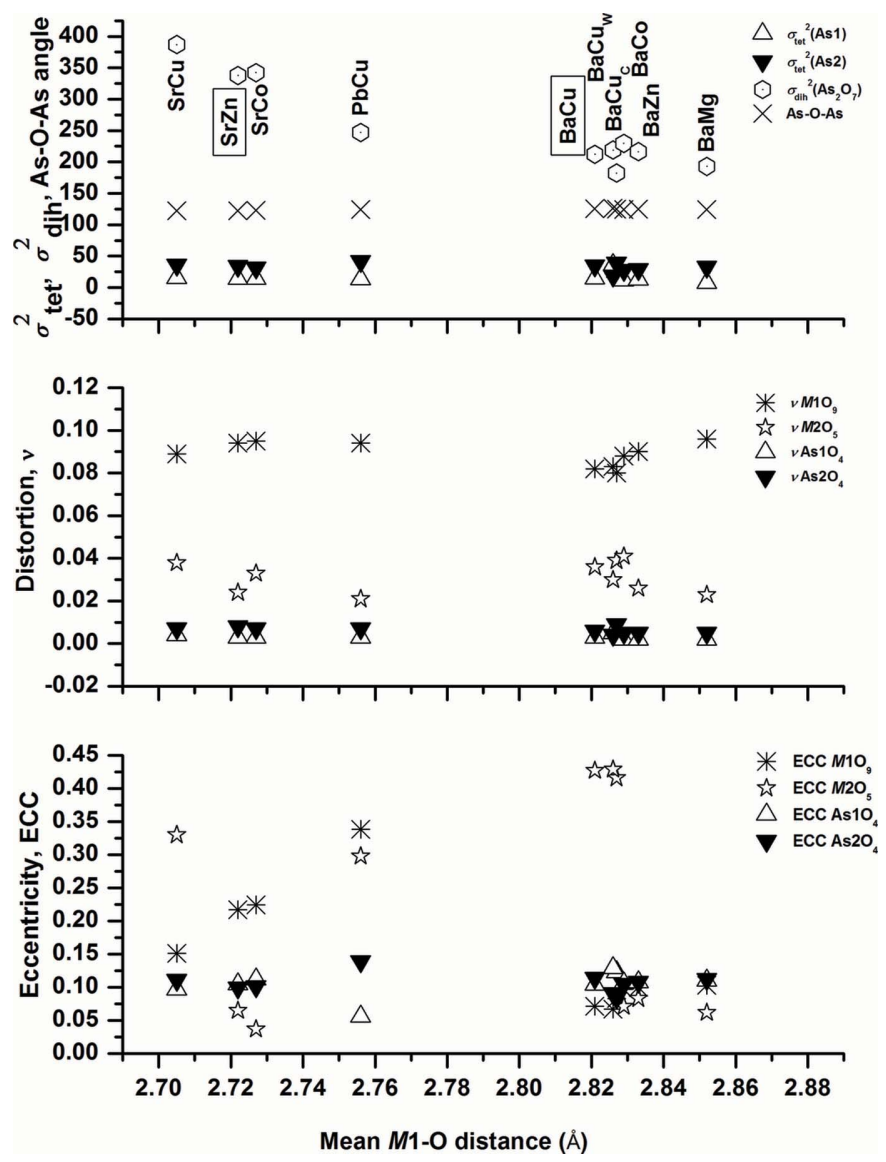

Figure 2

Distortion parameters versus average $\langle M 1-\mathrm{O}\rangle$ distances in $M 1^{\mathrm{II}} M 2^{\mathrm{II}} \mathrm{As}_{2} \mathrm{O}_{7}\left(M 1^{\mathrm{II}}=\mathrm{Sr}, \mathrm{Ba}, \mathrm{Pb} ; M 2^{\mathrm{II}}=\mathrm{Mg}, \mathrm{Co}, \mathrm{Cu}, \mathrm{Zn}\right)$ compounds. Framed symbols are for $\mathrm{SrZnAs}_{2} \mathrm{O}_{7}$ and $\mathrm{BaCuAs}_{2} \mathrm{O}_{7}$.
Table 4

Selected geometric parameters $\left(\AA,^{\circ}\right)$ for $\mathrm{BaCuAs}_{2} \mathrm{O}_{7}$.

\begin{tabular}{|c|c|c|c|}
\hline $\mathrm{Ba} 1-\mathrm{O} 2$ & $2.628(4)$ & As $1-O 1^{\mathrm{ii}}$ & $1.685(4)$ \\
\hline $\mathrm{Ba} 1-\mathrm{O} 2^{\mathrm{i}}$ & $2.683(5)$ & As1-O4 & 1.744 (4) \\
\hline $\mathrm{Ba} 1-\mathrm{O}^{\mathrm{ii}}$ & $2.764(4)$ & As $2-O 5^{\mathrm{v}}$ & $1.650(4)$ \\
\hline $\mathrm{Ba} 1-\mathrm{O} 7^{\mathrm{iii}}$ & $2.803(4)$ & As2-O6 & $1.685(4)$ \\
\hline $\mathrm{Ba} 1-\mathrm{O} 1^{\mathrm{iv}}$ & $2.803(4)$ & As2 $-\mathrm{O}^{\mathrm{iii}}$ & $1.698(4)$ \\
\hline $\mathrm{Ba} 1-\mathrm{O} 3^{\mathrm{i}}$ & $2.850(4)$ & As $2-O 4^{i}$ & $1.752(4)$ \\
\hline $\mathrm{Ba} 1-\mathrm{O}^{\mathrm{iv}}$ & $2.904(4)$ & $\mathrm{Cu} 1-\mathrm{O} 3$ & $1.932(4)$ \\
\hline $\mathrm{Ba} 1-\mathrm{O} 1^{\mathrm{ii}}$ & $2.938(4)$ & $\mathrm{Cu} 1-\mathrm{O} 7$ & $1.976(4)$ \\
\hline $\mathrm{Ba} 1-\mathrm{O} 5^{\mathrm{ii}}$ & $3.008(4)$ & $\mathrm{Cu} 1-\mathrm{O} 6$ & $1.983(4)$ \\
\hline $\mathrm{As} 1-\mathrm{O}^{\mathrm{ii}}$ & $1.654(4)$ & $\mathrm{Cu} 1-\mathrm{O} 1$ & $2.025(4)$ \\
\hline $\mathrm{As} 1-\mathrm{O} 3^{\mathrm{i}}$ & $1.663(4)$ & $\mathrm{Cu} 1-\mathrm{O} 5$ & $2.382(4)$ \\
\hline $\mathrm{O} 2-\mathrm{Ba} 1-\mathrm{O} 2^{\mathrm{i}}$ & $153.81(10)$ & $\mathrm{O} 6^{\mathrm{ii}}-\mathrm{Ba} 1-\mathrm{O} 5^{\mathrm{ii}}$ & $65.41(12)$ \\
\hline $\mathrm{O} 2-\mathrm{Ba} 1-\mathrm{O} 6^{\mathrm{ii}}$ & $125.38(13)$ & $\mathrm{O} 7^{\mathrm{iii}}-\mathrm{Ba} 1-\mathrm{O} 5^{\mathrm{ii}}$ & $63.22(12)$ \\
\hline $\mathrm{O} 2^{\mathrm{i}}-\mathrm{Ba} 1-\mathrm{O} 6^{\mathrm{ii}}$ & 76.68 (13) & $\mathrm{O} 1^{\mathrm{iv}}-\mathrm{Ba} 1-\mathrm{O} 5^{\mathrm{ii}}$ & $131.40(12)$ \\
\hline $\mathrm{O} 2-\mathrm{Ba}_{1}-\mathrm{O}^{\mathrm{iii}}$ & $104.89(14)$ & $\mathrm{O} 3^{\mathrm{i}}-\mathrm{Ba} 1-\mathrm{O} 5^{\mathrm{ii}}$ & $106.90(12)$ \\
\hline $\mathrm{O} 2^{\mathrm{i}}-\mathrm{Ba} 1-\mathrm{O} 7^{\mathrm{iii}}$ & $91.88(14)$ & $\mathrm{O} 5^{\mathrm{iv}}-\mathrm{Ba} 1-\mathrm{O} 5^{\mathrm{ii}}$ & $151.54(11)$ \\
\hline $\mathrm{O} 6^{\mathrm{ii}}-\mathrm{Ba} 1-\mathrm{O} 7^{\mathrm{iii}}$ & 80.15 (13) & $\mathrm{O} 1^{\mathrm{ii}}-\mathrm{Ba} 1-\mathrm{O} 5^{\mathrm{ii}}$ & $53.01(11)$ \\
\hline $\mathrm{O} 2-\mathrm{Ba} 1-\mathrm{O} 1^{\mathrm{iv}}$ & 90.97 (13) & $\mathrm{O} 2^{\mathrm{ii}}-\mathrm{As} 1-\mathrm{O}^{\mathrm{i}}$ & 115.9 (2) \\
\hline $\mathrm{O} 2^{\mathrm{i}}-\mathrm{Ba} 1-\mathrm{O} 1^{\mathrm{iv}}$ & $71.47(13)$ & $\mathrm{O} 2^{\mathrm{ii}}-\mathrm{As} 1-\mathrm{O} 1^{\mathrm{ii}}$ & $112.0(2)$ \\
\hline $\mathrm{O} 6^{\mathrm{ii}}-\mathrm{Ba} 1-\mathrm{O} 1^{\mathrm{iv}}$ & $142.42(13)$ & $\mathrm{O} 3^{\mathrm{i}}-\mathrm{As} 1-\mathrm{O} 1^{\mathrm{ii}}$ & $105.5(2)$ \\
\hline $\mathrm{O} 7^{\mathrm{iii}}-\mathrm{Ba} 1-\mathrm{O} 1^{\mathrm{iv}}$ & $81.45(12)$ & $\mathrm{O} 2^{\mathrm{ii}}-\mathrm{As} 1-\mathrm{O} 4$ & $108.5(2)$ \\
\hline $\mathrm{O} 2-\mathrm{Ba} 1-\mathrm{O} 3^{\mathrm{i}}$ & 96.66 (13) & $\mathrm{O}^{3}-\mathrm{As} 1-\mathrm{O} 4$ & $106.6(2)$ \\
\hline $\mathrm{O} 2^{\mathrm{i}}-\mathrm{Ba} 1-\mathrm{O} 3^{\mathrm{i}}$ & $76.64(13)$ & $\mathrm{O} 1^{\mathrm{ii}}-\mathrm{As} 1-\mathrm{O} 4$ & $108.0(2)$ \\
\hline $\mathrm{O}^{\mathrm{ii}}-\mathrm{Ba} 1-\mathrm{O}^{\mathrm{i}}$ & 70.14 (13) & $\mathrm{O} 5^{\mathrm{v}}-\mathrm{As} 2-\mathrm{O} 6$ & $115.4(2)$ \\
\hline $\mathrm{O} 7^{\mathrm{iii}}-\mathrm{Ba} 1-\mathrm{O}^{\mathrm{i}}$ & $149.83(13)$ & $\mathrm{O}^{\mathrm{v}}-\mathrm{As} 2-\mathrm{O}^{\mathrm{iii}}$ & $117.7(2)$ \\
\hline $\mathrm{O} 1^{\mathrm{iv}}-\mathrm{Ba} 1-\mathrm{O} 3^{\mathrm{i}}$ & $119.53(12)$ & $\mathrm{O} 6-\mathrm{As} 2-\mathrm{O}^{\mathrm{iii}}$ & $108.5(2)$ \\
\hline $\mathrm{O} 2-\mathrm{Ba} 1-\mathrm{O}^{\mathrm{iv}}$ & 83.98 (14) & $\mathrm{O} 5^{\mathrm{v}}-\mathrm{As} 2-\mathrm{O} 4^{\mathrm{i}}$ & $104.0(2)$ \\
\hline $\mathrm{O} 2^{\mathrm{i}}-\mathrm{Ba} 1-\mathrm{O}^{-\mathrm{iv}}$ & $70.11(13)$ & $\mathrm{O} 6-\mathrm{As} 2-\mathrm{O} 4^{\mathrm{i}}$ & $104.5(2)$ \\
\hline $\mathrm{O} 6^{\mathrm{ii}}-\mathrm{Ba} 1-\mathrm{O}^{\mathrm{iv}}$ & $129.47(13)$ & $\mathrm{O} 7^{\mathrm{iii}}-\mathrm{As} 2-\mathrm{O} 4^{\mathrm{i}}$ & $105.4(2)$ \\
\hline $\mathrm{O} 7^{\mathrm{iii}}-\mathrm{Ba} 1-\mathrm{O} 5^{\mathrm{iv}}$ & $136.35(12)$ & $\mathrm{O} 3-\mathrm{Cu} 1-\mathrm{O} 7$ & 87.90 (19) \\
\hline $\mathrm{O} 1^{\mathrm{iv}}-\mathrm{Ba} 1-\mathrm{O} 5^{\mathrm{iv}}$ & $55.40(12)$ & $\mathrm{O} 3-\mathrm{Cu} 1-\mathrm{O} 6$ & $158.73(19)$ \\
\hline $\mathrm{O} 3^{\mathrm{i}}-\mathrm{Ba} 1-\mathrm{O}^{\mathrm{iv}}$ & $65.93(12)$ & $\mathrm{O} 7-\mathrm{Cu} 1-\mathrm{O} 6$ & 89.01 (18) \\
\hline $\mathrm{O} 2-\mathrm{Ba} 1-\mathrm{O} 1^{\mathrm{ii}}$ & $70.08(13)$ & $\mathrm{O} 3-\mathrm{Cu} 1-\mathrm{O} 1$ & $91.48(18)$ \\
\hline $\mathrm{O} 2^{\mathrm{i}}-\mathrm{Ba} 1-\mathrm{O} 1^{\mathrm{ii}}$ & $121.67(12)$ & $\mathrm{O} 7-\mathrm{Cu} 1-\mathrm{O} 1$ & $172.97(17)$ \\
\hline $\mathrm{O} 6^{\mathrm{ii}}-\mathrm{Ba} 1-\mathrm{O} 1^{\mathrm{ii}}$ & $58.97(12)$ & $\mathrm{O} 6-\mathrm{Cu} 1-\mathrm{O} 1$ & 89.03 (18) \\
\hline $\mathrm{O} 7^{\mathrm{iii}}-\mathrm{Ba} 1-\mathrm{O} 1^{\mathrm{ii}}$ & $113.44(12)$ & $\mathrm{O} 3-\mathrm{Cu} 1-\mathrm{O} 5$ & 109.54 (17) \\
\hline $\mathrm{O} 1^{\mathrm{iv}}-\mathrm{Ba} 1-\mathrm{O} 1^{\mathrm{ii}}$ & $158.02(6)$ & $\mathrm{O} 7-\mathrm{Cu} 1-\mathrm{O} 5$ & $113.23(17)$ \\
\hline $\mathrm{O} 3^{\mathrm{i}}-\mathrm{Ba} 1-\mathrm{O} 1^{\mathrm{ii}}$ & $54.80(12)$ & $\mathrm{O} 6-\mathrm{Cu} 1-\mathrm{O} 5$ & 90.99 (17) \\
\hline $\mathrm{O} 5^{\mathrm{iv}}-\mathrm{Ba} 1-\mathrm{O} 1^{\mathrm{ii}}$ & $109.76(12)$ & $\mathrm{O} 1-\mathrm{Cu} 1-\mathrm{O} 5$ & $73.56(16)$ \\
\hline $\mathrm{O} 2-\mathrm{Ba} 1-\mathrm{O}^{\mathrm{ii}}$ & $69.15(13)$ & $\mathrm{As} 1-\mathrm{O} 4-\mathrm{As} 2^{\mathrm{vi}}$ & $125.3(2)$ \\
\hline $\mathrm{O} 2^{\mathrm{i}}-\mathrm{Ba} 1-\mathrm{O} 5^{\mathrm{ii}}$ & $137.03(13)$ & & \\
\hline
\end{tabular}

Symmetry codes: (i) $-x+\frac{1}{2}, y+\frac{1}{2},-z+\frac{1}{2}$; (ii) $x-1, y, z$; (iii) $-x+1,-y+1,-z$; (iv) $-x+\frac{3}{2}, y+\frac{1}{2},-z+\frac{1}{2} ;$ (v) $-x+2,-y+1,-z ;\left(\right.$ vi) $-x+\frac{1}{2}, y-\frac{1}{2},-z+\frac{1}{2}$.

noted that some authors (Pertlik, 1986; Horng \& Wang, 1994) describe $M 1$ as eight-coordinated, taking into account only $\mathrm{O}$ atoms at distances less than $3 \AA$. In order to observe $M 1$ cations in a similar environment and to compare the distortion parameters in different diarsenates, the same $\mathrm{CN}=9$ for all M1 cations was assumed. The bond-valence calculations (Brown, 1996; Wills, 2010) show that the $\mathrm{Sr}$ atom in $\mathrm{SrZnAs}_{2} \mathrm{O}_{7}$ is in good agreement with its formal oxidation state $\left(\Sigma v_{i j}=1.99\right.$ v.u. $)$ if $\mathrm{CN}=9$, although the longest $\mathrm{Sr}-\mathrm{O}$ bond length [3.268 (3) A] contributes only $2 \%$ to the bondvalence sum. The sum of the bond valences of the $\mathrm{Ba}$ atom in $\mathrm{BaCuAs}_{2} \mathrm{O}_{7}$ suggests a slight oversaturation if all nine ligands are considered $\left(\Sigma v_{i j}=2.2\right.$ v.u. $)$, but it is the same as the value calculated for $\mathrm{BaCoAs}_{2} \mathrm{O}_{7}$ (Mihajlović et al., 2004). The longest $\mathrm{Ba}-\mathrm{O}$ distance $[3.008(4) \AA]$ contributes $6 \%$ to the bond-valence sum.

As a measure for polyhedron distortion of the $\mathrm{AsO}_{4}$ tetrahedra, the $M 1 \mathrm{O}_{9}$ tricapped trigonal prisms and the $M 2 \mathrm{O}_{5}$ square pyramids, the volume-based eccentricity (ECC) and 
Table 5

Geometrical characteristics of the coordination polyhedra for $M 1^{\mathrm{II}} M 2^{\mathrm{II}} \mathrm{As}_{2} \mathrm{O}_{7}$ compounds $\left(M 1=\mathrm{Ba}^{2+}, \mathrm{Sr}^{2+}, \mathrm{Pb}^{2+}, \mathrm{Ca}^{2+}, M 2=\mathrm{Cu}^{2+}, \mathrm{Mg}^{2+}, \mathrm{Zn}^{2+}, \mathrm{Ni}^{2+}\right.$, $\left.\mathrm{Co}^{2+}, \mathrm{Cr}^{2+}\right)$.

\begin{tabular}{|c|c|c|c|c|c|c|c|}
\hline Compound & $\begin{array}{l}\text { Central atom } \\
(\mathrm{CN})\end{array}$ & $\begin{array}{l}\text { Average } M-\mathrm{O} \\
\text { bond distance }(\AA)\end{array}$ & $\begin{array}{l}\text { Radius of a fitted } \\
\text { circumscribed } \\
\text { sphere }(\AA)\end{array}$ & $\begin{array}{l}\text { Sum of ionic radii } \\
\text { for the bonds* }(\AA)\end{array}$ & $\begin{array}{l}\text { Volume-based } \\
\text { ECCs }\end{array}$ & $\begin{array}{l}\text { Volume-based } \\
\text { distortion, } v\end{array}$ & $\begin{array}{l}\sigma_{\text {tet }}^{2}, \lambda(\mathrm{As} 1), \\
\sigma_{\text {tet }}^{2}, \lambda(\mathrm{As} 2), \\
\text { As } 1-\mathrm{O}_{\mathrm{b}}-\mathrm{As} 2 \\
\text { angle }\left(^{\circ}\right), \sigma_{\mathrm{dih}}^{2}\end{array}$ \\
\hline \multirow[t]{4}{*}{$\mathrm{SrCuAs}_{2} \mathrm{O}_{7}^{1}$} & As1 (4) & $1.683 \pm 0.037$ & 1.683 & 1.715 & 0.097 & 0.004 & $15.73,1.0038$ \\
\hline & As2 (4) & $1.695 \pm 0.041$ & 1.692 & 1.715 & 0.111 & 0.007 & $35.89,1.0089$ \\
\hline & $\operatorname{Sr}(9)$ & $2.705 \pm 0.175$ & 2.699 & 2.69 & 0.151 & 0.089 & $122(2)$ \\
\hline & $\mathrm{Cu}(5)$ & $2.035 \pm 0.133$ & 2.026 & 2.03 & 0.330 & 0.038 & 386.89 \\
\hline \multirow{4}{*}{$\mathrm{SrCoAs}_{2} \mathrm{O}_{7}^{2}$} & As1 (4) & $1.682 \pm 0.043$ & 1.680 & 1.715 & 0.112 & 0.003 & $14.25,1.0035$ \\
\hline & As2 (4) & $1.693 \pm 0.037$ & 1.691 & 1.715 & 0.101 & 0.007 & $31.73,1.0078$ \\
\hline & $\operatorname{Sr}(9)$ & $2.727 \pm 0.235$ & 2.718 & 2.69 & 0.224 & 0.095 & $122.7(3)$ \\
\hline & Co $(5)$ & $2.063 \pm 0.068$ & 2.061 & 2.05 & 0.037 & 0.033 & 342.20 \\
\hline \multirow{4}{*}{$\mathrm{SrZnAs}_{2} \mathrm{O}_{7}{ }^{3}$} & As1 (4) & $1.685 \pm 0.040$ & 1.684 & 1.715 & 0.105 & 0.003 & $14.24,1.0032$ \\
\hline & As2 (4) & $1.695 \pm 0.037$ & 1.692 & 1.715 & 0.099 & 0.008 & $34.26,1.0085$ \\
\hline & $\operatorname{Sr}(9)$ & $2.722 \pm 0.231$ & 2.714 & 2.69 & 0.217 & 0.094 & 122.39 (18) \\
\hline & $\mathrm{Zn}(5)$ & $2.054 \pm 0.075$ & 2.053 & 2.06 & 0.065 & 0.024 & 337.71 \\
\hline \multirow[t]{4}{*}{$\mathrm{PbCuAs}_{2} \mathrm{O}_{7}{ }^{4}$} & As1 (4) & $1.686 \pm 0.021$ & 1.686 & 1.715 & 0.056 & 0.003 & $13.77,1.0033$ \\
\hline & As2 (4) & $1.701 \pm 0.052$ & 1.697 & 1.715 & 0.139 & 0.007 & $41.62,1.0103$ \\
\hline & $\mathrm{Pb}(9)$ & $2.756 \pm 0.278$ & 2.744 & 2.73 & 0.338 & 0.094 & $124.46(8)$ \\
\hline & $\mathrm{Cu}(5)$ & $2.033 \pm 0.121$ & 2.023 & 2.03 & 0.298 & 0.021 & 246.99 \\
\hline \multirow[t]{4}{*}{$\mathrm{BaCuAs}_{2} \mathrm{O}_{7}^{\mathbf{1}}$} & As1 (4) & $1.690 \pm 0.047$ & 1.688 & 1.715 & 0.123 & 0.005 & $25.04,1.0037$ \\
\hline & As2 (4) & $1.695 \pm 0.031$ & 1.692 & 1.715 & 0.084 & 0.009 & $39.06,1.0095$ \\
\hline & $\mathrm{Ba}(9)$ & $2.827 \pm 0.126$ & 2.820 & 2.85 & 0.083 & 0.080 & $123(3)$ \\
\hline & $\mathrm{Cu}(5)$ & $2.047 \pm 0.175$ & 2.038 & 2.03 & 0.416 & 0.039 & 182.37 \\
\hline \multirow[t]{4}{*}{$\mathrm{BaCuAs}_{2} \mathrm{O}_{7}{ }^{3}$} & As1 (4) & $1.686 \pm 0.039$ & 1.685 & 1.715 & 0.104 & 0.003 & $14.96,1.0039$ \\
\hline & As2 (4) & $1.696 \pm 0.042$ & 1.693 & 1.715 & 0.114 & 0.006 & $34.80,1.0082$ \\
\hline & $\mathrm{Ba}(9)$ & $2.821 \pm 0.120$ & 2.816 & 2.85 & 0.071 & 0.082 & 125.32 (19) \\
\hline & $\mathrm{Cu}(5)$ & $2.057 \pm 0.183$ & 2.049 & 2.03 & 0.427 & 0.036 & 212.30 \\
\hline \multirow[t]{4}{*}{$\mathrm{BaCuAs}_{2} \mathrm{O}_{7}{ }^{\mathbf{5}}$} & As1 (4) & $1.695 \pm 0.049$ & 1.691 & 1.715 & 0.129 & 0.005 & $36.04,1.0087$ \\
\hline & As2 (4) & $1.682 \pm 0.035$ & 1.681 & 1.715 & 0.091 & 0.004 & $18.57,1.0033$ \\
\hline & $\mathrm{Ba}(9)$ & $2.826 \pm 0.120$ & 2.822 & 2.85 & 0.067 & 0.083 & $125.9(3)$ \\
\hline & $\mathrm{Cu}(5)$ & $2.061 \pm 0.185$ & 2.053 & 2.03 & 0.429 & 0.030 & 219.00 \\
\hline \multirow[t]{4}{*}{$\mathrm{BaMgAs}_{2} \mathrm{O}_{7}{ }^{6}$} & As1 (4) & $1.685 \pm 0.042$ & 1.685 & 1.715 & 0.110 & 0.002 & $7.96,1.0022$ \\
\hline & As2 (4) & $1.694 \pm 0.042$ & 1.692 & 1.715 & 0.112 & 0.005 & $32.70,1.0069$ \\
\hline & $\mathrm{Ba}(9)$ & $2.852 \pm 0.178$ & 2.848 & 2.85 & 0.103 & 0.096 & $125.0(2)$ \\
\hline & $\operatorname{Mg}(5)$ & $2.049 \pm 0.038$ & 2.047 & 2.04 & 0.062 & 0.023 & 192.99 \\
\hline \multirow[t]{4}{*}{$\mathrm{BaCoAs}_{2} \mathrm{O}_{7}{ }^{6}$} & As1 (4) & $1.686 \pm 0.041$ & 1.685 & 1.715 & 0.106 & 0.002 & $11.91,1.003$ \\
\hline & As2 (4) & $1.696 \pm 0.039$ & 1.693 & 1.715 & 0.105 & 0.005 & $27.55,1.007$ \\
\hline & $\mathrm{Ba}(9)$ & $2.829 \pm 0.154$ & 2.824 & 2.85 & 0.097 & 0.088 & $125.0(2)$ \\
\hline & Co (5) & $2.069 \pm 0.056$ & 2.066 & 2.05 & 0.071 & 0.041 & 229.64 \\
\hline \multirow[t]{4}{*}{$\mathrm{BaZnAs}_{2} \mathrm{O}_{7}{ }^{7}$} & As1 (4) & $1.687 \pm 0.041$ & 1.686 & 1.715 & 0.108 & 0.002 & $12.71,1.0028$ \\
\hline & As2 (4) & $1.690 \pm 0.040$ & 1.688 & 1.715 & 0.108 & 0.005 & $28.97,1.0073$ \\
\hline & $\mathrm{Ba}(9)$ & $2.833 \pm 0.164$ & 2.829 & 2.85 & 0.100 & 0.090 & $124.67(14)$ \\
\hline & $\mathrm{Zn}(5)$ & $2.061 \pm 0.054$ & 2.059 & 2.06 & 0.083 & 0.026 & 216.59 \\
\hline
\end{tabular}

Notes: (*) calculated from effective ionic radii (Shannon, 1976); (\#) $\sigma_{\mathrm{dih}}^{2}=\Sigma\left(\theta_{i}-60\right)^{2} / 3(i=1,2,3) . \quad$ References: (1) Chen \& Wang (1996); (2) Horng \& Wang (1994); (3) this work; (4) Pertlik (1986); (5) Wardojo \& Hwu (1995); (6) Mihajlović et al. (2004); (7) Đorđević (2008).

the volume-based distortion ( $v$ ) were calculated using the program IVTON (Balić-Žunić \& Makovicky, 1996; Makovicky
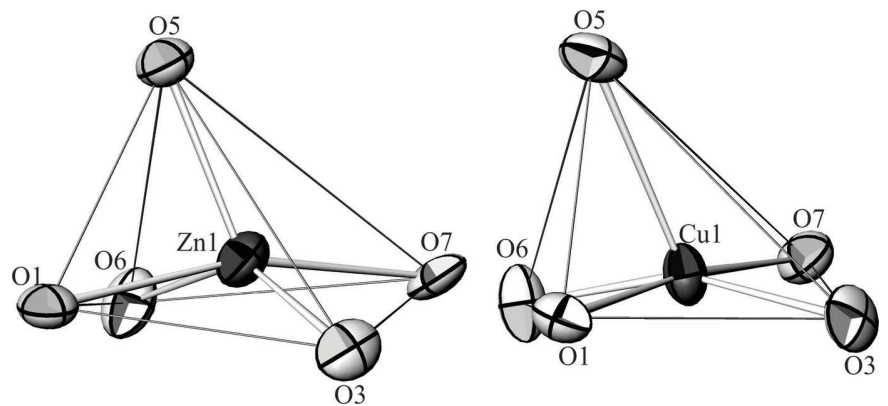

Figure 3

Displacement ellipsoid plot ( $90 \%$ probability level) of the $\mathrm{M}_{2} \mathrm{O}_{5}$ square pyramids in $\mathrm{SrZnAs}_{2} \mathrm{O}_{7}$ and $\mathrm{BaCuAs}_{2} \mathrm{O}_{7}$, showing the distortion and the atomic numbering schemes.
\& Balić-Žunić, 1998). The ECC is a measure of the displacement of the central atom from the centroid of the coordination polyhedron defined as the point for which the variance of squares of the central cation-to-corner ligand distances is at a minimum. The $v$ parameter is an overall measure of polyhedral distortion which can be defined as the regularity of distribution of the ligand atoms irrespective of the position of the central atom in the polyhedron. Unsurprisingly, in all the $M 1^{\mathrm{II}} M 2^{\mathrm{II}} \mathrm{As}_{2} \mathrm{O}_{7}$ compounds, the $\mathrm{As}_{10} \mathrm{O}_{4}$ and $\mathrm{As} 2 \mathrm{O}_{4}$ tetrahedra are less distorted than the $M 1 \mathrm{O}_{9}$ and $M 2 \mathrm{O}_{5}$ polyhedra. The mean quadratic elongation, $\lambda$, and the tetrahedral angle variance, $\sigma_{\text {tet }}^{2}$, indicate that the $\mathrm{As} 2 \mathrm{O}_{4}$ tetrahedra are a little more distorted than $\mathrm{As}_{10}$ (Table 5 and Fig. 2). The low ECC (0.056-0.123 for $\mathrm{As} 1 \mathrm{O}_{4}$ and $0.084-0.139$ for $\left.\mathrm{As}_{2} \mathrm{O}_{4}\right)$ and low $v$ (0.002-0.005 for $\mathrm{As} 1 \mathrm{O}_{4}$ and 0.004-0.009 for $\mathrm{As}_{2} \mathrm{O}_{4}$ ) reflect the regularity of the tetrahedra (Fig. 2). The bridging bond angles 
As1 $-\mathrm{O}_{\mathrm{b}}-$ As2 range from $122.4(2)$ to $125.3(2)^{\circ}$ and depend slightly on the $M 1$ cation size (Table 5), i.e. the angles become a little wider as the $M 1$ cation becomes larger. In contrast, the values of the dihedral angle variances depend greatly on the $M 1$ cation size and the variance becomes smaller as the $M 1$ cations become larger, suggesting that a smaller $M 1$ cation causes a larger dihedral angle deviation from the ideal value of $60^{\circ}$. The ECC $(0.037-0.083)$ of the $M 2^{\mathrm{II}}$ cations in the $\mathrm{ZnO}_{5}$, $\mathrm{CoO}_{5}$ and $\mathrm{MgO}_{5}$ pyramids are low, indicating that these $\mathrm{M2}^{\mathrm{II}}$ cations are located close to the centroid of the $M 2 \mathrm{O}_{5}$ pyramids. The ECC (0.298-0.429) clearly increases with the incorporation of $\mathrm{Cu}^{\mathrm{II}}$ cations in the $\mathrm{M} 2$ position (Table 5 and Fig. 2) leading to the almost uniform $\mathrm{Cu}-\mathrm{O}$ basal distances and a longer $\mathrm{Cu}-\mathrm{O}$ apical distance, which is in agreement with the Jahn-Teller effect of the $\mathrm{Cu}^{\mathrm{II}}$ cation (Fig. 3). The $M 2$ sites in three $\mathrm{BaCuAs}_{2} \mathrm{O}_{7}$ compounds have the highest eccentricity of all. $\mathrm{PbCuAs}_{2} \mathrm{O}_{7}$ displays a more eccentric $\mathrm{Pb}_{10}$, polyhedron than the $\mathrm{Sr}-$ and Ba-containing $M 1^{\mathrm{II}} M 2^{\mathrm{II}} \mathrm{As}_{2} \mathrm{O}_{7}$ compounds, because of its inert electron pair. The $v$ values $(0.021-0.041)$ of the $M 2 \mathrm{O}_{5}$ polyhedra are, as expected, higher than for the $\mathrm{As} 1 \mathrm{O}_{4}$ and $\mathrm{As}_{2} \mathrm{O}_{4}$ tetrahedra, but lower than for the $M 1 \mathrm{O}_{9}$ coordination polyhedra: $v\left(\mathrm{BaO}_{9}\right)$ $=0.080-0.096(\langle 0.082\rangle), v\left(\mathrm{SrO}_{9}\right)=0.089-0.095(\langle 0.094\rangle)$ and $v\left(\mathrm{PbO}_{9}\right)=0.094$ (Table 5). These values indicate a slightly lower distortion of the $M 1 \mathrm{O}_{9}$ coordination polyhedra for larger $M 1^{\mathrm{II}}$ cations. It is interesting that $v$ has the largest values in compounds containing $\mathrm{Co}$, i.e. for the $\mathrm{CoO}_{5}$ pyramid in $\mathrm{BaCoAs}_{2} \mathrm{O}_{7}$ and for the $\mathrm{SrO}_{9}$ polyhedra in $\mathrm{SrCoAs}_{2} \mathrm{O}_{7}$. In $\mathrm{SrZnAs}_{2} \mathrm{O}_{7}$ and $\mathrm{BaCuAs}_{2} \mathrm{O}_{7}$, although both $M 1^{\mathrm{II}}$ cations are moved from the corresponding centroids $[\mathrm{ECC}(\mathrm{Sr})=0.217$ and $\mathrm{ECC}(\mathrm{Ba})=0.071]$, judging by the $v$ values $[v(\mathrm{Sr})=0.094$ and $v(\mathrm{Ba})=0.082]$, the coordinated $\mathrm{O}$ atoms form quite regular polyhedra.

As already mentioned, the lattice parameters obtained for $\mathrm{BaCuAs}_{2} \mathrm{O}_{7}$ deviate somewhat from the values reported earlier by Wardojo \& Hwu (1995) and Chen \& Wang (1996). The slight increase in unit-cell volume of approximately $4 \AA^{3}$ [632.7 (2), 634.9 (2) and $636.6(6) \AA^{3}$ for $\mathrm{BaCu}_{\mathrm{C}}, \mathrm{BaCu}$ and $\mathrm{BaCu}_{\mathrm{w}}$, respectively; Table 5 and Fig. 2] reflects the increase of the dihedral angle variances, $\sigma_{\mathrm{dih}}^{2}$, which are $182.37,212.30$ and $219.00^{\circ}$ for $\mathrm{BaCu}_{\mathrm{C}}, \mathrm{BaCu}$ and $\mathrm{BaCu}_{\mathrm{W}}$, respectively. This indicates that the smallest $\mathrm{As}_{2} \mathrm{O}_{7}$ angular deviation in $\mathrm{BaCuAs}_{2} \mathrm{O}_{7}$ corresponds to the smallest unit cell (Table 5 and Fig. 2). Contrary to the dihedral angle variances, the tetrahedral angle variances, $\sigma_{\text {tet }}^{2}$, vary over relatively small intervals, viz. 14.96-25.04 and 34.80-39.06 ${ }^{\circ}$, which confirms the statement that the $\mathrm{As}_{2} \mathrm{O}_{7}$ group is adjustable with regard to the bonding requirements. The other geometrical characteristics of the coordination polyhedra are nearly the same.

\section{Acknowledgements}

The authors gratefully acknowledge financial support by the Ministry of Education, Science and Technological Development of the Republic of Serbia (grant No. III45007) and the Austrian Science Foundation (FWF) (grant No. V203-N19).
The authors are thankful to Professor Dr T. Balić-Žunić for helping with the IVTON calculations.

\section{References}

Adams, R. D., Layland, R. C. \& Payen, C. (1995). Polyhedron, 14, 3473-3480.

Alaoui El Belghiti, A., Boukhari, A. \& Holt, E. M. (1991). Acta Cryst. C47, 473-477.

Altomare, A., Burla, M. C., Camalli, M., Cascarano, G. L., Giacovazzo, C., Guagliardi, A., Moliterni, A. G. G., Polidori, G. \& Spagna, R. (1999). J. Appl. Cryst. 32, 115-119.

Balić-Zunić, T. \& Makovicky, E. (1996). Acta Cryst. B52, 78-81.

Brown, I. D. (1996). J. Appl. Cryst. 29, 479-480.

Brown, I. D. \& Calvo, C. (1970). J. Solid State Chem. 1, 173-179.

Calvo, C. (1968). Inorg. Chem. 7, 1345-1351.

Chen, T. C. \& Wang, S. L. (1996). J. Solid State Chem. 121, 350-355.

Đorđević, T. (2008). Acta Cryst. E64, i57.

Dowty, E. (2000). ATOMS for Windows. Shape Software, Kingsport, Tennessee, USA.

Edhokkar, F., Hadrich, A., Graia, M. \& Mhiri, T. (2012). 2012 IOP Conf. Ser. Mater. Sci. Eng. 28, 012017.

El Bali, B., Boukhari, A., Aride, J., Maass, K., Wald, D., Glaum, R. \& Abraham, F. (2001). Solid State Sci. 3, 669-676.

Elmarzouki, A., Boukhari, A., Holt, E. M. \& Berrada, A. (1994). J. Alloys Compd, 204, 127-131.

Elmarzouki, A., Boukhari, A., Holt, E. M. \& Berrada, A. (1995). J. Alloys Compd, 227, 125-130.

Faggiani, R. \& Calvo, C. (1976). Can. J. Chem. 54, 3319-3324.

Farrugia, L. J. (2012). J. Appl. Cryst. 45, 849-854.

Hazen, R. M. \& Finger, L. W. (1982). In Comparative Crystal Chemistry, p. 86 and Appendix III. Chichester: Wiley.

Höppe, H. A., Daub, M. \& Bröhmer, M. C. (2007). Chem. Mater. 19, 6358-6362.

Horchani-Naifer, K. \& Férid, M. (2007). Acta Cryst. E63, i33-i34.

Horng, J.-C. \& Wang, S.-L. (1994). Acta Cryst. C50, 488-490.

ICSD (2014). Inorganic Crystal Structure Database. FIZ-Karlsruhe, Germany. http://icsd.fiz-karlsruhe.de/icsd/.

Kolitsch, U. (2004). Z. Kristallogr. New Cryst. Struct. 219, 207-208.

Le Meins, J.-M. \& Courbion, G. (1999). Acta Cryst. C55, 481-483.

Maass, K. \& Glaum, R. (2000). Acta Cryst. C56, 404-406.

Makovicky, E. \& Balić-Žunić, T. (1998). Acta Cryst. B54, 766-773.

Marzouki, R., Guesmi, A., Zid, M. F. \& Driss, A. (2012). Cryst. Struct. Theor. Appl. 1, 68-73.

Mihajlović, T. \& Effenberger, H. (2006). Z. Kristallogr. 221, 770-781.

Mihajlović, T., Kolitsch, U. \& Effenberger, H. (2004). J. Alloys Compd, 379, 103-109.

Moqine, A., Boukhari, A., Elammari, L. \& Durand, J. (1993). J. Solid State Chem. 107, 368-372.

Murashova, E. V., Velikodnyi, Y. A. \& Trunov, V. K. (1991). Zh. Neorg. Khim. 36, 851-854.

Nonius (2002). COLLECT. Nonius BV, Delft, The Netherlands.

Otwinowski, Z., Borek, D., Majewski, W. \& Minor, W. (2003). Acta Cryst. A59, 228-234.

Otwinowski, Z. \& Minor, W. (1997). Methods in Enzymology, Vol. 276, Macromolecular Crystallography, Part A, edited by C. W. Carter Jr \& R. M. Sweet, pp. 307-326. New York: Academic Press. Ouerfelli, N., Guesmi, A., Molinié, P., Mazza, D., Zid, M. F. \& Driss, A. (2007). J. Solid State Chem. 180, 2942-2949.

Pertlik, F. (1986). Monatsh. Chem. 117, 1343-1348.

Riou, D. \& Goreaud, M. (1990). Acta Cryst. C46, 1191-1193.

Riou, D. \& Raveau, B. (1991). Acta Cryst. C47, 1708-1709.

Robinson, K., Gibbs, G. V. \& Ribbe, P. H. (1971). Science, 172, 567570.

Rousse, G., Rodríguez-Carvajal, J., Wurm, C. \& Masquelier, C. (2013). Phys. Rev. B, 88, 214433.

Schwendtner, K. \& Kolitsch, U. (2004). Acta Cryst. C60, i79-i83. 
Shannon, R. D. (1976). Acta Cryst. A32, 751-767.

Sheldrick, G. M. (2015). Acta Cryst. C71, 3-8.

Spek, A. L. (2009). Acta Cryst. D65, 148-155.

Tahiri, A. A., Bali, B. E., Lachkar, M., Ouarsal, R. \& Zavalij, P. Y. (2002). Acta Cryst. E58, i9-i11.

Wardojo, T. A. \& Hwu, S. J. (1995). J. Solid State Chem. 118, 280-284.
Weil, M., Đorđević, T., Lengauer, C. L. \& Kolitsch, U. (2009). Solid State Sci. 11, 2111-2117.

Westrip, S. P. (2010). J. Appl. Cryst. 43, 920-925.

Wills, A. S. (2010). VaList. http://www.CCP14.ac.uk.

Yuan, J. L., Zeng, X. Y., Zhao, J. T., Zhang, Z. J., Chen, H. H. \& Zhang, G. B. (2007). J. Solid State Chem. 180, 3310-3316. 


\section{supporting information}

Acta Cryst. (2015). C71, 330-337 [doi:10.1107/S2053229615005616]

\section{An investigation of polyhedral deformation in two mixed-metal diarsenates: $\mathrm{SrZnAs}_{2} \mathrm{O}_{7}$ and $\mathrm{BaCuAs}_{2} \mathrm{O}_{7}$}

\section{Sabina Kovač, Ljiljana Karanović and Tamara Đorđević}

\section{Computing details}

For both compounds, data collection: COLLECT (Nonius, 2002); cell refinement: SCALEPACK (Otwinowski \& Minor, 1997); data reduction: DENZO-SMN (Otwinowski et al., 2003); program(s) used to solve structure: SIR97 (Altomare et al., 1999). Program(s) used to refine structure: SHELXL2013 (Sheldrick, 2015), WinGX (Farrugia, 2012) and PLATON (Spek, 2009) for SrZnAs2O7; SHELXL2013 (Sheldrick, 2015) and WinGX (Farrugia, 2012) for BaCuAs2O7. For both compounds, molecular graphics: ATOMS (Dowty, 2000); software used to prepare material for publication: publCIF (Westrip, 2010).

\section{(SrZnAs2O7) Strontium zinc diarsenic heptaoxide}

Crystal data

$\mathrm{SrZnAs}_{2} \mathrm{O}_{7}$

$M_{r}=414.85$

Monoclinic, $P 2{ }_{1} / n$

$a=5.5114(11) \AA$

$b=8.3524(17) \AA$

$c=13.106(3) \AA$

$\beta=91.01(3)^{\circ}$

$V=603.2(2) \AA^{3}$

Data collection

Nonius KappaCCD diffractometer

Radiation source: fine-focus sealed X-ray tube $\varphi$ and $\omega$ scans

Absorption correction: multi-scan (Otwinowski \& Minor, 1997)

$T_{\min }=0.668, T_{\max }=1.000$

7055 measured reflections

Refinement

Refinement on $F^{2}$

Least-squares matrix: full

$R\left[F^{2}>2 \sigma\left(F^{2}\right)\right]=0.032$

$w R\left(F^{2}\right)=0.074$

$S=1.04$

1834 reflections

100 parameters

0 restraints

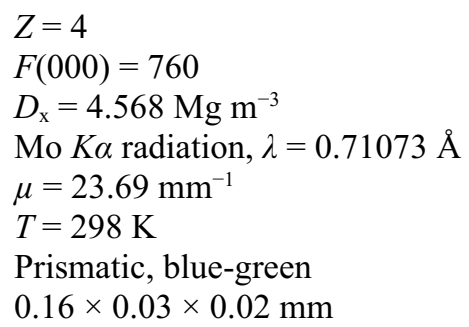

1835 independent reflections 1555 reflections with $I>2 \sigma(I)$

$R_{\text {int }}=0.062$

$\theta_{\max }=30.5^{\circ}, \theta_{\min }=2.9^{\circ}$

$h=-7 \rightarrow 7$

$k=-11 \rightarrow 11$

$l=-18 \rightarrow 18$

Primary atom site location: structure-invariant direct methods

Secondary atom site location: difference Fourier map

$w=1 /\left[\sigma^{2}\left(F_{\mathrm{o}}^{2}\right)+(0.0401 P)^{2}+1.0702 P\right]$ where $P=\left(F_{\mathrm{o}}^{2}+2 F_{\mathrm{c}}^{2}\right) / 3$

$(\Delta / \sigma)_{\max }<0.001$

$\Delta \rho_{\max }=1.35 \mathrm{e}^{-3}$

$\Delta \rho_{\min }=-1.20 \mathrm{e} \AA^{-3}$ 


\section{Special details}

Geometry. All e.s.d.'s (except the e.s.d. in the dihedral angle between two 1.s. planes) are estimated using the full covariance matrix. The cell e.s.d.'s are taken into account individually in the estimation of e.s.d.'s in distances, angles and torsion angles; correlations between e.s.d.'s in cell parameters are only used when they are defined by crystal symmetry. An approximate (isotropic) treatment of cell e.s.d.'s is used for estimating e.s.d.'s involving l.s. planes.

Fractional atomic coordinates and isotropic or equivalent isotropic displacement parameters $\left(\AA^{2}\right)$

\begin{tabular}{lllll}
\hline & $x$ & $y$ & $z$ & $U_{\text {iso }} * U_{\text {eq }}$ \\
\hline Sr1 & $0.20949(7)$ & $0.67034(5)$ & $0.21859(3)$ & $0.01119(11)$ \\
As1 & $-0.24486(7)$ & $0.47658(5)$ & $0.33857(3)$ & $0.00824(11)$ \\
As2 & $0.67278(7)$ & $0.69498(5)$ & $0.01457(3)$ & $0.00820(11)$ \\
Zn1 & $0.83776(9)$ & $0.35654(6)$ & $0.10963(4)$ & $0.01061(12)$ \\
O1 & $0.9575(5)$ & $0.3826(4)$ & $0.2663(2)$ & $0.0117(6)$ \\
O2 & $0.4720(5)$ & $0.4537(4)$ & $0.2972(3)$ & $0.0153(7)$ \\
O3 & $0.6522(6)$ & $0.1665(4)$ & $0.1521(3)$ & $0.0124(6)$ \\
O4 & $-0.2204(6)$ & $0.3987(4)$ & $0.4618(2)$ & $0.0132(6)$ \\
O5 & $1.1990(5)$ & $0.3270(4)$ & $0.0989(2)$ & $0.0119(6)$ \\
O6 & $0.8294(5)$ & $0.5986(4)$ & $0.1072(2)$ & $0.0128(6)$ \\
O7 & $0.6245(5)$ & $0.3445(4)$ & $-0.0251(2)$ & $0.0112(6)$ \\
\hline
\end{tabular}

Atomic displacement parameters $\left(\AA^{2}\right)$

\begin{tabular}{lllllll}
\hline & $U^{11}$ & $U^{22}$ & $U^{33}$ & $U^{12}$ & $U^{13}$ & $U^{23}$ \\
\hline Sr1 & $0.00753(19)$ & $0.0120(2)$ & $0.0140(2)$ & $-0.00041(14)$ & $-0.00140(15)$ & $0.00130(15)$ \\
As1 & $0.00647(19)$ & $0.0078(2)$ & $0.0104(2)$ & $0.00031(14)$ & $-0.00097(15)$ & $-0.00028(15)$ \\
As2 & $0.0062(2)$ & $0.0096(2)$ & $0.0088(2)$ & $0.00015(14)$ & $-0.00062(15)$ & $-0.00011(15)$ \\
Zn1 & $0.0082(2)$ & $0.0101(2)$ & $0.0134(3)$ & $-0.00048(18)$ & $-0.00143(18)$ & $0.00016(19)$ \\
O1 & $0.0082(14)$ & $0.0150(15)$ & $0.0118(15)$ & $0.0032(11)$ & $0.0010(12)$ & $-0.0016(12)$ \\
O2 & $0.0066(14)$ & $0.0137(16)$ & $0.0253(19)$ & $-0.0001(11)$ & $-0.0055(13)$ & $-0.0007(13)$ \\
O3 & $0.0118(15)$ & $0.0063(14)$ & $0.0189(16)$ & $0.0007(11)$ & $0.0004(12)$ & $0.0015(12)$ \\
O4 & $0.0183(15)$ & $0.0094(15)$ & $0.0118(15)$ & $0.0033(12)$ & $-0.0005(12)$ & $0.0006(12)$ \\
O5 & $0.0077(14)$ & $0.0174(16)$ & $0.0106(15)$ & $0.0000(11)$ & $0.0002(11)$ & $-0.0002(12)$ \\
O6 & $0.0117(15)$ & $0.0104(15)$ & $0.0161(16)$ & $0.0004(11)$ & $-0.0053(12)$ & $0.0015(12)$ \\
O7 & $0.0044(13)$ & $0.0157(16)$ & $0.0136(15)$ & $-0.0011(11)$ & $-0.0001(11)$ & $0.0015(13)$ \\
& & & & & &
\end{tabular}

Geometric parameters $\left(\hat{A},{ }^{\circ}\right)$

\begin{tabular}{llll}
\hline $\mathrm{Sr} 1-\mathrm{O} 2$ & $2.525(3)$ & $\mathrm{Zn} 1-\mathrm{O} 3$ & $1.973(3)$ \\
$\mathrm{Sr} 1-\mathrm{O} 1^{\mathrm{i}}$ & $2.557(3)$ & $\mathrm{Zn} 1-\mathrm{O} 5$ & $2.013(3)$ \\
$\mathrm{Sr} 1-\mathrm{O} 2^{\mathrm{ii}}$ & $2.576(3)$ & $\mathrm{Zn} 1-\mathrm{O} 6$ & $2.023(3)$ \\
$\mathrm{Sr} 1-\mathrm{O}^{\text {iii }}$ & $2.602(3)$ & $\mathrm{Zn} 1-\mathrm{O} 7$ & $2.106(3)$ \\
$\mathrm{Sr} 1-\mathrm{O} 3^{\text {ii }}$ & $2.640(3)$ & $\mathrm{Zn} 1-\mathrm{O} 1$ & $2.157(3)$ \\
$\mathrm{Sr} 1-\mathrm{O} 7^{\mathrm{iv}}$ & $2.714(3)$ & $\mathrm{Zn} 1-\mathrm{Sr} 1^{\mathrm{vii}}$ & $3.6059(9)$ \\
$\mathrm{Sr} 1-\mathrm{O} 5^{\mathrm{i}}$ & $2.765(3)$ & $\mathrm{Zn} 1-\mathrm{Sr} 1^{\text {viii }}$ & $3.6762(11)$ \\
$\mathrm{Sr} 1-\mathrm{O} 1^{\mathrm{iii}}$ & $2.851(3)$ & $\mathrm{O} 1-\mathrm{As} 1^{\mathrm{vii}}$ & $1.671(3)$ \\
$\mathrm{Sr} 1-\mathrm{O} 5^{\mathrm{iii}}$ & $3.268(3)$ & $\mathrm{O} 1-\mathrm{Sr} 1^{\text {viii }}$ & $2.557(3)$ \\
$\mathrm{Sr} 1-\mathrm{As} 1$ & $3.3913(9)$ & $\mathrm{O} 1-\mathrm{Sr} 1^{\text {vii }}$ & $2.851(3)$
\end{tabular}




\begin{tabular}{|c|c|c|c|}
\hline $\mathrm{Sr} 1-\mathrm{Zn} 1^{\mathrm{iii}}$ & $3.6059(9)$ & $\mathrm{O} 2-\mathrm{As} 1^{\mathrm{vii}}$ & $1.654(3)$ \\
\hline $\mathrm{Sr} 1-\mathrm{Zn} 1^{\mathrm{i}}$ & $3.6762(11)$ & $\mathrm{O} 2-\mathrm{Sr} 1^{\mathrm{ix}}$ & $2.576(3)$ \\
\hline $\mathrm{As} 1-\mathrm{O} 2^{\mathrm{iii}}$ & $1.654(3)$ & $\mathrm{O} 3-\mathrm{As} 1^{\mathrm{ix}}$ & $1.670(3)$ \\
\hline $\mathrm{As} 1-\mathrm{O} 3^{\mathrm{ii}}$ & $1.670(3)$ & $\mathrm{O} 3-\mathrm{Sr}^{\mathrm{ix}}$ & $2.640(3)$ \\
\hline As1-O $1^{\mathrm{iii}}$ & $1.671(3)$ & $\mathrm{O} 4-\mathrm{As} 2^{\mathrm{ix}}$ & $1.749(3)$ \\
\hline As $1-\mathrm{O} 4$ & $1.744(3)$ & $\mathrm{O} 5-\mathrm{As} 2^{\mathrm{vi}}$ & $1.669(3)$ \\
\hline $\mathrm{As} 1-\mathrm{Sr}^{\mathrm{v}}$ & $3.6869(8)$ & $\mathrm{O} 5-\mathrm{Sr} 1^{\text {viii }}$ & $2.765(3)$ \\
\hline As1-Sr1iii & $3.7374(10)$ & $\mathrm{O} 5-\mathrm{Sr} 1^{\mathrm{vii}}$ & $3.268(3)$ \\
\hline As2-O5 $5^{\mathrm{vi}}$ & $1.669(3)$ & $\mathrm{O} 6-\mathrm{Sr} 1^{\mathrm{vii}}$ & $2.602(3)$ \\
\hline As $2-O 7^{\text {iv }}$ & $1.679(3)$ & $\mathrm{O} 7-\mathrm{As} 2^{\mathrm{iv}}$ & $1.679(3)$ \\
\hline As $2-O 6$ & $1.682(3)$ & $\mathrm{O} 7-\mathrm{Sr}^{\mathrm{iv}}$ & $2.714(3)$ \\
\hline $\mathrm{As} 2-\mathrm{O} 4^{\mathrm{ii}}$ & $1.749(3)$ & & \\
\hline $\mathrm{O} 2-\mathrm{Sr} 1-\mathrm{O} 1^{\mathrm{i}}$ & $93.44(10)$ & 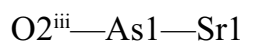 & $127.11(12)$ \\
\hline $\mathrm{O} 2-\mathrm{Sr} 1-\mathrm{O} 2^{\mathrm{ii}}$ & $155.50(7)$ & $\mathrm{O} 3^{\mathrm{ii}}-\mathrm{As} 1-\mathrm{Sr} 1$ & $49.74(11)$ \\
\hline $\mathrm{O} 1^{\mathrm{i}}-\mathrm{Sr} 1-\mathrm{O} 2^{\mathrm{ii}}$ & $69.30(10)$ & 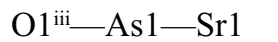 & $57.05(11)$ \\
\hline $\mathrm{O} 2-\mathrm{Sr} 1-\mathrm{O} 6^{\mathrm{iii}}$ & $120.91(10)$ & $\mathrm{O} 4-\mathrm{As} 1-\mathrm{Sr} 1$ & $124.17(11)$ \\
\hline $\mathrm{O} 1^{\mathrm{i}}-\mathrm{Sr} 1-\mathrm{O}^{\mathrm{iii}}$ & $140.59(10)$ & 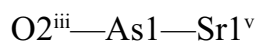 & $37.37(11)$ \\
\hline $\mathrm{O} 2^{\mathrm{ii}}-\mathrm{Sr} 1-\mathrm{O}^{6 i i}$ & $81.88(10)$ & $\mathrm{O} 3^{\mathrm{ii}}-\mathrm{As} 1-\mathrm{Sr}^{\mathrm{v}}$ & $152.14(11)$ \\
\hline $\mathrm{O} 2-\mathrm{Sr} 1-\mathrm{O}{ }^{\mathrm{ii}}$ & $99.39(10)$ & 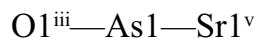 & $91.54(11)$ \\
\hline $\mathrm{O} 1^{\mathrm{i}}-\mathrm{Sr} 1-\mathrm{O} 3^{\mathrm{ii}}$ & $120.53(10)$ & $\mathrm{O} 4-\mathrm{As} 1-\mathrm{Sr} 1^{\mathrm{v}}$ & 88.37 (11) \\
\hline 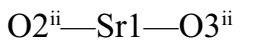 & $76.47(10)$ & $\mathrm{Sr} 1-\mathrm{As} 1-\mathrm{Sr} 1^{\mathrm{v}}$ & $138.830(16)$ \\
\hline $\mathrm{O}^{\mathrm{iii}}-\mathrm{Sr} 1-\mathrm{O}^{\mathrm{ii}}$ & $75.40(10)$ & $\mathrm{O} 2^{\mathrm{iii}}-\mathrm{As} 1-\mathrm{Sr} 1^{\mathrm{iii}}$ & $33.36(11)$ \\
\hline $\mathrm{O} 2-\mathrm{Sr} 1-\mathrm{O}^{\mathrm{iv}}$ & $98.52(11)$ & 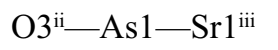 & 82.08 (11) \\
\hline $\mathrm{O} 1^{\mathrm{i}}-\mathrm{Sr} 1-\mathrm{O}^{\mathrm{iv}}$ & 81.41 (10) & $\mathrm{O} 1^{\mathrm{iii}}-\mathrm{As} 1-\mathrm{Sr} 1^{\mathrm{iii}}$ & $120.33(11)$ \\
\hline $\mathrm{O} 2^{\mathrm{ii}}-\mathrm{Sr} 1-\mathrm{O} 7^{\mathrm{iv}}$ & $95.96(10)$ & $\mathrm{O} 4-\mathrm{As} 1-\mathrm{Sr} 1^{\mathrm{iii}}$ & $126.83(11)$ \\
\hline $\mathrm{O}^{\mathrm{iii}}-\mathrm{Sr} 1-\mathrm{O}^{\mathrm{iv}}$ & $75.32(9)$ & $\operatorname{Sr} 1-\mathrm{As} 1-\mathrm{Sr} 1^{1 \mathrm{iii}}$ & $101.16(2)$ \\
\hline $\mathrm{O}^{\mathrm{ii}}-\mathrm{Sr} 1-\mathrm{O}^{\mathrm{iv}}$ & $150.51(10)$ & $\operatorname{Sr} 1^{\mathrm{v}}-\mathrm{As} 1-\mathrm{Sr} 1^{\mathrm{iii}}$ & $70.374(18)$ \\
\hline $\mathrm{O} 2-\mathrm{Sr} 1-\mathrm{O}^{\mathrm{i}}$ & $83.80(10)$ & $\mathrm{O} 5^{\mathrm{vi}}-\mathrm{As} 2-\mathrm{O}^{\mathrm{iv}}$ & $118.74(15)$ \\
\hline $\mathrm{O} 1^{\mathrm{i}}-\mathrm{Sr} 1-\mathrm{O} 5^{\mathrm{i}}$ & $59.01(10)$ & $\mathrm{O} 5^{\mathrm{vi}}-\mathrm{As} 2-\mathrm{O} 6$ & $111.75(16)$ \\
\hline 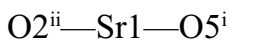 & $72.49(10)$ & $\mathrm{O} 7^{\mathrm{iv}}-\mathrm{As} 2-\mathrm{O} 6$ & $109.62(15)$ \\
\hline $\mathrm{O}^{\mathrm{iii}}-\mathrm{Sr} 1-\mathrm{O} 5^{\mathrm{i}}$ & $136.65(10)$ & $\mathrm{O} 5^{\mathrm{vi}}-\mathrm{As} 2-\mathrm{O} 4^{\mathrm{ii}}$ & $101.56(15)$ \\
\hline 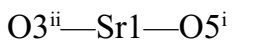 & $65.08(9)$ & $\mathrm{O} 7^{\mathrm{iv}}-\mathrm{As} 2-\mathrm{O} 4^{\mathrm{ii}}$ & $108.69(15)$ \\
\hline $\mathrm{O} 7^{\mathrm{iv}}-\mathrm{Sr} 1-\mathrm{O} 5^{\mathrm{i}}$ & $140.40(9)$ & $\mathrm{O} 6-\mathrm{As} 2-\mathrm{O} 4^{\mathrm{ii}}$ & $105.33(15)$ \\
\hline $\mathrm{O} 2-\mathrm{Sr} 1-\mathrm{O} 1^{\mathrm{iii}}$ & $65.43(9)$ & $\mathrm{O} 5^{\mathrm{vi}}-\mathrm{As} 2-\mathrm{Sr} 1$ & $159.71(11)$ \\
\hline 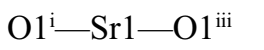 & $156.84(6)$ & $\mathrm{O} 7^{\mathrm{iv}}-\mathrm{As} 2-\mathrm{Sr} 1$ & $41.43(11)$ \\
\hline $\mathrm{O} 2^{\mathrm{ii}}-\mathrm{Sr} 1-\mathrm{O} 1^{\mathrm{iii}}$ & $127.02(9)$ & $\mathrm{O} 6-\mathrm{As} 2-\mathrm{Sr} 1$ & $78.79(11)$ \\
\hline $\mathrm{O}^{\mathrm{iii}}-\mathrm{Sr} 1-\mathrm{O} 1^{\mathrm{iii}}$ & $62.52(9)$ & $\mathrm{O} 4^{\mathrm{ii}}-\mathrm{As} 2-\mathrm{Sr} 1$ & $91.67(11)$ \\
\hline $\mathrm{O} 3^{\mathrm{ii}}-\mathrm{Sr} 1-\mathrm{O} 1^{\mathrm{iii}}$ & $58.16(9)$ & $\mathrm{O} 3-\mathrm{Zn} 1-\mathrm{O} 5$ & $116.04(13)$ \\
\hline $\mathrm{O} 7^{\mathrm{iv}}-\mathrm{Sr} 1-\mathrm{O} 1^{\mathrm{iii}}$ & $109.88(9)$ & $\mathrm{O} 3-\mathrm{Zn} 1-\mathrm{O} 6$ & $142.82(13)$ \\
\hline $\mathrm{O} 5^{\mathrm{i}}-\mathrm{Sr} 1-\mathrm{O} 1^{\mathrm{iii}}$ & $106.95(9)$ & $\mathrm{O} 5-\mathrm{Zn} 1-\mathrm{O} 6$ & $98.26(13)$ \\
\hline $\mathrm{O} 2-\mathrm{Sr} 1-\mathrm{O} 5^{\mathrm{iii}}$ & $64.66(10)$ & $\mathrm{O} 3-\mathrm{Zn} 1-\mathrm{O} 7$ & 85.04 (13) \\
\hline $\mathrm{O} 1^{\mathrm{i}}-\mathrm{Sr} 1-\mathrm{O} 5^{\mathrm{iii}}$ & $130.69(9)$ & $\mathrm{O} 5-\mathrm{Zn} 1-\mathrm{O} 7$ & $118.29(12)$ \\
\hline $\mathrm{O} 2^{\mathrm{ii}}-\mathrm{Sr} 1-\mathrm{O} 5^{\mathrm{iii}}$ & $139.81(9)$ & $\mathrm{O} 6-\mathrm{Zn} 1-\mathrm{O} 7$ & $91.26(12)$ \\
\hline $\mathrm{O}^{\mathrm{iii}}-\mathrm{Sr} 1-\mathrm{O}^{\mathrm{iii}}$ & $61.41(9)$ & $\mathrm{O} 3-\mathrm{Zn} 1-\mathrm{O} 1$ & 87.94 (13) \\
\hline $\mathrm{O} 3^{\mathrm{ii}}-\mathrm{Sr} 1-\mathrm{O} 5^{\mathrm{iii}}$ & $106.87(9)$ & $\mathrm{O} 5-\mathrm{Zn} 1-\mathrm{O} 1$ & $78.02(12)$ \\
\hline $\mathrm{O}^{1 \mathrm{iv}}-\mathrm{Sr} 1-\mathrm{O}^{6 \mathrm{iii}}$ & $60.98(9)$ & $\mathrm{O} 6-\mathrm{Zn} 1-\mathrm{O} 1$ & $85.46(12)$ \\
\hline $\mathrm{O} 5^{\mathrm{i}}-\mathrm{Sr} 1-\mathrm{O} 5^{\mathrm{iii}}$ & $146.28(9)$ & $\mathrm{O} 7-\mathrm{Zn} 1-\mathrm{O} 1$ & $163.69(12)$ \\
\hline
\end{tabular}




\begin{tabular}{|c|c|c|c|}
\hline 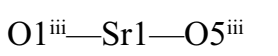 & $50.28(8)$ & $\mathrm{O} 3-\mathrm{Zn} 1-\mathrm{Sr} 1^{\mathrm{vii}}$ & $140.05(10)$ \\
\hline $\mathrm{O} 2-\mathrm{Sr} 1-\mathrm{As} 1$ & $83.76(7)$ & $\mathrm{O} 5-\mathrm{Zn} 1-\mathrm{Sr} 1^{\mathrm{vii}}$ & $63.96(9)$ \\
\hline $\mathrm{O} 1 \mathrm{i}-\mathrm{Sr} 1-\mathrm{As} 1$ & $146.09(7)$ & $\mathrm{O} 6-\mathrm{Zn} 1-\mathrm{Sr} 1^{\mathrm{vii}}$ & $44.96(8)$ \\
\hline $\mathrm{O} 2^{\mathrm{ii}}-\mathrm{Sr} 1-\mathrm{As} 1$ & $100.76(7)$ & $\mathrm{O} 7-\mathrm{Zn} 1-\mathrm{Sr} 1^{\mathrm{vii}}$ & $132.18(9)$ \\
\hline $\mathrm{O}^{\mathrm{iii}}-\mathrm{Sr} 1-\mathrm{As} 1$ & $63.68(7)$ & $\mathrm{O} 1-\mathrm{Zn} 1-\mathrm{Sr} 1^{\mathrm{vii}}$ & $52.23(9)$ \\
\hline $\mathrm{O} 3^{\mathrm{ii}}-\mathrm{Sr} 1-\mathrm{As} 1$ & $28.87(7)$ & $\mathrm{O} 3-\mathrm{Zn} 1-\mathrm{Sr} 1^{\mathrm{viii}}$ & $80.60(9)$ \\
\hline $\mathrm{O}^{\mathrm{iv}}-\mathrm{Sr} 1-\mathrm{As} 1$ & $132.48(7)$ & $\mathrm{O} 5-\mathrm{Zn} 1-\mathrm{Sr} 1^{\text {viii }}$ & $47.89(9)$ \\
\hline $\mathrm{O}^{\mathrm{i}}-\mathrm{Sr} 1-\mathrm{As} 1$ & $87.12(7)$ & $\mathrm{O} 6-\mathrm{Zn} 1-\mathrm{Sr} 1^{\mathrm{viii}}$ & $116.59(9)$ \\
\hline $\mathrm{O} 1^{\mathrm{iii}}-\mathrm{Sr} 1-\mathrm{As} 1$ & $29.47(6)$ & $\mathrm{O} 7-\mathrm{Zn} 1-\mathrm{Sr} 1^{\text {viii }}$ & $148.87(9)$ \\
\hline 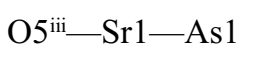 & $78.27(6)$ & $\mathrm{O} 1-\mathrm{Zn} 1-\mathrm{Sr} 1^{\mathrm{viii}}$ & $42.84(8)$ \\
\hline $\mathrm{O} 2-\mathrm{Sr} 1-\mathrm{Zn} 1^{\mathrm{iii}}$ & $87.59(8)$ & $\mathrm{Sr} 1^{\mathrm{vii}}-\mathrm{Zn} 1-\mathrm{Sr} 1^{\text {viii }}$ & $71.96(2)$ \\
\hline 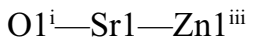 & $159.49(7)$ & As $1{ }^{\mathrm{vii}}-\mathrm{O} 1-\mathrm{Zn} 1$ & $113.01(15)$ \\
\hline $\mathrm{O} 2^{\mathrm{ii}}-\mathrm{Sr} 1-\mathrm{Zn} 1^{\mathrm{iii}}$ & $114.76(7)$ & As1 $1^{\text {vii }}-\mathrm{O} 1-\mathrm{Sr} 1^{\mathrm{viii}}$ & $140.09(17)$ \\
\hline $\mathrm{O}^{\mathrm{iii}}-\mathrm{Sr} 1-\mathrm{Zn} 1^{\mathrm{iii}}$ & $33.32(7)$ & $\mathrm{Zn} 1-\mathrm{O} 1-\mathrm{Sr}^{\mathrm{viii}}$ & $102.17(12)$ \\
\hline $\mathrm{O} 3^{\mathrm{ii}}-\mathrm{Sr} 1-\mathrm{Zn} 1^{\mathrm{iii}}$ & $79.36(7)$ & $\mathrm{As} 1^{\mathrm{vii}}-\mathrm{O} 1-\mathrm{Sr}^{\mathrm{vii}}$ & $93.48(13)$ \\
\hline $\mathrm{O} 7^{\mathrm{iv}}-\mathrm{Sr} 1-\mathrm{Zn} 1^{\mathrm{iii}}$ & $78.19(7)$ & $\mathrm{Zn} 1-\mathrm{O} 1-\mathrm{Sr}^{\mathrm{vii}}$ & $91.04(11)$ \\
\hline 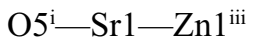 & $141.29(7)$ & $\mathrm{Sr} 1^{\mathrm{viii}}-\mathrm{O} 1-\mathrm{Sr}^{\mathrm{vii}}$ & $104.45(10)$ \\
\hline 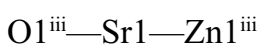 & $36.73(6)$ & 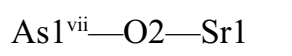 & $125.53(17)$ \\
\hline $\mathrm{O} 5^{\mathrm{iii}}-\mathrm{Sr} 1-\mathrm{Zn} 1^{\mathrm{iii}}$ & $33.60(5)$ & 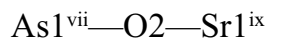 & $119.69(16)$ \\
\hline As1-Sr1-Zn1 ${ }^{\mathrm{iii}}$ & $54.392(17)$ & $\mathrm{Sr} 1-\mathrm{O} 2-\mathrm{Sr} 1^{\mathrm{ix}}$ & $114.01(11)$ \\
\hline $\mathrm{O} 2-\mathrm{Sr} 1-\mathrm{Zn} 1^{\mathrm{i}}$ & $71.08(8)$ & $\mathrm{As} 1^{\mathrm{ix}}-\mathrm{O} 3-\mathrm{Zn} 1$ & $128.72(18)$ \\
\hline 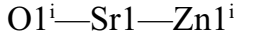 & $34.99(7)$ & $\mathrm{As} 1^{\mathrm{ix}}-\mathrm{O} 3-\mathrm{Sr} 1^{\mathrm{ix}}$ & $101.39(14)$ \\
\hline $\mathrm{O} 2^{\mathrm{ii}}-\mathrm{Sr} 1-\mathrm{Zn} 1^{\mathrm{i}}$ & $85.35(7)$ & $\mathrm{Zn} 1-\mathrm{O} 3-\mathrm{Sr} 1^{\mathrm{ix}}$ & $124.90(14)$ \\
\hline $\mathrm{O}^{\mathrm{iii}}-\mathrm{Sr} 1-\mathrm{Zn} 1^{\mathrm{i}}$ & $166.41(7)$ & $\mathrm{As} 1-\mathrm{O} 4-\mathrm{As} 2^{\mathrm{ix}}$ & $122.39(18)$ \\
\hline $\mathrm{O}{ }^{\mathrm{ii}}-\mathrm{Sr} 1-\mathrm{Zn} 1^{\mathrm{i}}$ & $97.12(7)$ & As2 $2^{\mathrm{vi}}-\mathrm{O} 5-\mathrm{Zn} 1$ & $120.70(17)$ \\
\hline $\mathrm{O}^{\mathrm{iv}}-\mathrm{Sr} 1-\mathrm{Zn} 1^{\mathrm{i}}$ & $110.80(7)$ & $\mathrm{As} 2^{\mathrm{vi}}-\mathrm{O} 5-\mathrm{Sr} 1^{\mathrm{viii}}$ & $130.19(16)$ \\
\hline 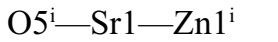 & $32.70(6)$ & $\mathrm{Zn} 1-\mathrm{O} 5-\mathrm{Sr} 1^{\mathrm{viii}}$ & $99.40(12)$ \\
\hline 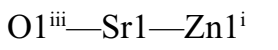 & $123.39(6)$ & $\mathrm{As} 2^{\mathrm{vi}}-\mathrm{O} 5-\mathrm{Sr} 1^{\mathrm{vii}}$ & $121.35(15)$ \\
\hline 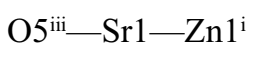 & $132.15(5)$ & $\mathrm{Zn} 1-\mathrm{O} 5-\mathrm{Sr} 1^{\mathrm{vii}}$ & $82.44(10)$ \\
\hline As 1 - $\mathrm{Sr} 1-\mathrm{Zn} 1^{\mathrm{i}}$ & $114.63(2)$ & $\mathrm{Sr} 1^{\mathrm{viii}}-\mathrm{O} 5-\mathrm{Sr} 1^{\mathrm{vii}}$ & $89.94(9)$ \\
\hline $\mathrm{Zn} 1^{\mathrm{iii}}-\mathrm{Sr} 1-\mathrm{Zn} 1^{\mathrm{i}}$ & $157.632(18)$ & As2-O6-Zn1 & $120.07(17)$ \\
\hline 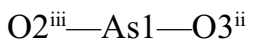 & $114.79(16)$ & As2-O6-Sr1 $1^{\mathrm{vi}}$ & $133.84(17)$ \\
\hline 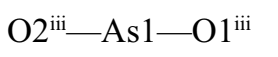 & $113.26(16)$ & $\mathrm{Zn} 1-\mathrm{O} 6-\mathrm{Sr} 1^{\mathrm{vii}}$ & $101.73(12)$ \\
\hline $\mathrm{O} 3^{\mathrm{ii}}-\mathrm{As} 1-\mathrm{O} 1^{\mathrm{iii}}$ & $106.36(16)$ & $\mathrm{As} 2^{\mathrm{iv}}-\mathrm{O} 7-\mathrm{Zn} 1$ & $118.14(17)$ \\
\hline $\mathrm{O} 2^{\mathrm{iii}}-\mathrm{As} 1-\mathrm{O} 4$ & $108.51(17)$ & $\mathrm{As} 2^{\mathrm{iv}}-\mathrm{O} 7-\mathrm{Sr} 1^{\mathrm{iv}}$ & $114.40(15)$ \\
\hline $\mathrm{O} 3^{\mathrm{ii}}-\mathrm{As} 1-\mathrm{O} 4$ & $105.51(16)$ & $\mathrm{Zn} 1-\mathrm{O} 7-\mathrm{Sr} 1^{\mathrm{iv}}$ & $126.37(12)$ \\
\hline $\mathrm{O} 11^{\mathrm{iii}}-\mathrm{As} 1-\mathrm{O} 4$ & $107.95(15)$ & & \\
\hline
\end{tabular}

Symmetry codes: (i) $-x+3 / 2, y+1 / 2,-z+1 / 2$; (ii) $-x+1 / 2, y+1 / 2,-z+1 / 2$; (iii) $x-1, y, z$; (iv) $-x+1,-y+1,-z$; (v) $-x-1 / 2, y-1 / 2,-z+1 / 2$; (vi) $-x+2,-y+1,-z$; (vii) $x+1, y, z$; (viii) $-x+3 / 2, y-1 / 2,-z+1 / 2$; (ix) $-x+1 / 2, y-1 / 2,-z+1 / 2$.

\section{(BaCuAs2O7) Barium copper diarsenic heptaoxide}

Crystal data

$\mathrm{BaCuAs}_{2} \mathrm{O}_{7}$

$M_{r}=462.72$

Monoclinic, $P 2_{1} / n$

$a=5.7343(11) \AA$

$b=8.4722(17) \AA$

$c=13.071(3) \AA$ $\beta=91.12(3)^{\circ}$

$V=634.9(2) \AA^{3}$

$Z=4$

$F(000)=828$

$D_{\mathrm{x}}=4.841 \mathrm{Mg} \mathrm{m}^{-3}$

Mo $K \alpha$ radiation, $\lambda=0.71073 \AA$ 
$\mu=19.84 \mathrm{~mm}^{-1}$

$T=298 \mathrm{~K}$

\section{Data collection}

Nonius KappaCCD diffractometer

Radiation source: fine-focus sealed X-ray tube $\varphi$ and $\omega$ scans

Absorption correction: multi-scan (Otwinowski \& Minor, 1997)

$T_{\min }=0.807, T_{\max }=1.000$

4991 measured reflections

\section{Refinement}

Refinement on $F^{2}$

Least-squares matrix: full

$R\left[F^{2}>2 \sigma\left(F^{2}\right)\right]=0.025$

$w R\left(F^{2}\right)=0.059$

$S=1.20$

1297 reflections

101 parameters

0 restraints
Prismatic, blue-green

$0.06 \times 0.03 \times 0.02 \mathrm{~mm}$

1298 independent reflections

1232 reflections with $I>2 \sigma(I)$

$R_{\text {int }}=0.025$

$\theta_{\text {max }}=26.4^{\circ}, \theta_{\text {min }}=2.9^{\circ}$

$h=-7 \rightarrow 7$

$k=-10 \rightarrow 10$

$l=-16 \rightarrow 16$

\section{Special details}

Geometry. All e.s.d.'s (except the e.s.d. in the dihedral angle between two 1.s. planes) are estimated using the full covariance matrix. The cell e.s.d.'s are taken into account individually in the estimation of e.s.d.'s in distances, angles and torsion angles; correlations between e.s.d.'s in cell parameters are only used when they are defined by crystal symmetry. An approximate (isotropic) treatment of cell e.s.d.'s is used for estimating e.s.d.'s involving l.s. planes.

Fractional atomic coordinates and isotropic or equivalent isotropic displacement parameters $\left(\AA^{2}\right)$

\begin{tabular}{lllll}
\hline & $x$ & $y$ & $z$ & $U_{\text {iso }} * / U_{\text {eq }}$ \\
\hline Ba1 & $0.21641(6)$ & $0.65177(4)$ & $0.21373(3)$ & $0.01078(13)$ \\
As1 & $-0.25303(10)$ & $0.46629(7)$ & $0.34475(4)$ & $0.00811(16)$ \\
As2 & $0.67164(10)$ & $0.68332(7)$ & $0.00787(4)$ & $0.00849(16)$ \\
Cu1 & $0.78989(13)$ & $0.35893(8)$ & $0.11961(6)$ & $0.01007(18)$ \\
O1 & $0.9278(7)$ & $0.3766(5)$ & $0.2630(3)$ & $0.0115(8)$ \\
O2 & $0.4698(7)$ & $0.4399(5)$ & $0.3119(4)$ & $0.0170(9)$ \\
O3 & $0.6632(8)$ & $0.1528(5)$ & $0.1488(3)$ & $0.0138(9)$ \\
O4 & $-0.2048(8)$ & $0.3849(5)$ & $0.4659(3)$ & $0.0126(9)$ \\
O5 & $1.2020(8)$ & $0.3402(5)$ & $0.1034(3)$ & $0.0142(9)$ \\
O6 & $0.8041(7)$ & $0.5920(5)$ & $0.1077(3)$ & $0.0135(9)$ \\
O7 & $0.6194(7)$ & $0.3516(5)$ & $-0.0132(3)$ & $0.0124(9)$ \\
\hline
\end{tabular}

Atomic displacement parameters $\left(\AA^{2}\right)$

\begin{tabular}{lllllll}
\hline & $U^{11}$ & $U^{22}$ & $U^{33}$ & $U^{12}$ & $U^{13}$ & $U^{23}$ \\
\hline Ba1 & $0.0117(2)$ & $0.00914(19)$ & $0.0115(2)$ & $-0.00027(13)$ & $-0.00038(13)$ & $0.00043(13)$ \\
As1 & $0.0103(3)$ & $0.0060(3)$ & $0.0079(3)$ & $0.0003(2)$ & $-0.0006(2)$ & $-0.0002(2)$ \\
As2 & $0.0104(3)$ & $0.0082(3)$ & $0.0069(3)$ & $-0.0001(2)$ & $0.0000(2)$ & $0.0000(2)$ \\
Cu1 & $0.0144(4)$ & $0.0067(4)$ & $0.0090(4)$ & $-0.0007(3)$ & $-0.0022(3)$ & $0.0006(3)$ \\
O1 & $0.012(2)$ & $0.011(2)$ & $0.011(2)$ & $0.0012(15)$ & $0.0017(16)$ & $-0.0003(16)$
\end{tabular}




\begin{tabular}{lllllll} 
O2 & $0.010(2)$ & $0.016(2)$ & $0.024(2)$ & $0.0012(17)$ & $-0.0035(18)$ & $0.0004(19)$ \\
O3 & $0.019(2)$ & $0.007(2)$ & $0.016(2)$ & $0.0013(17)$ & $0.0022(18)$ & $0.0016(17)$ \\
O4 & $0.022(2)$ & $0.0067(19)$ & $0.009(2)$ & $0.0017(17)$ & $-0.0015(17)$ & $0.0017(16)$ \\
O5 & $0.016(2)$ & $0.014(2)$ & $0.013(2)$ & $0.0026(17)$ & $0.0058(17)$ & $0.0006(17)$ \\
O6 & $0.016(2)$ & $0.009(2)$ & $0.015(2)$ & $-0.0005(16)$ & $-0.0050(17)$ & $-0.0001(17)$ \\
O7 & $0.010(2)$ & $0.015(2)$ & $0.012(2)$ & $-0.0029(16)$ & $-0.0003(17)$ & $0.0005(16)$ \\
\hline
\end{tabular}

Geometric parameters $\left(\AA,{ }^{\circ}\right)$

\begin{tabular}{|c|c|c|c|}
\hline $\mathrm{Ba} 1-\mathrm{O} 2$ & $2.628(4)$ & $\mathrm{Cu} 1-\mathrm{O} 3$ & $1.932(4)$ \\
\hline $\mathrm{Ba} 1-\mathrm{O} 2^{\mathrm{i}}$ & $2.683(5)$ & $\mathrm{Cu} 1-\mathrm{O} 7$ & $1.976(4)$ \\
\hline $\mathrm{Ba} 1-\mathrm{O}^{\mathrm{ii}}$ & $2.764(4)$ & $\mathrm{Cu} 1-\mathrm{O} 6$ & $1.983(4)$ \\
\hline $\mathrm{Ba} 1-\mathrm{O}^{\mathrm{iii}}$ & $2.803(4)$ & $\mathrm{Cu} 1-\mathrm{O} 1$ & $2.025(4)$ \\
\hline $\mathrm{Ba} 1-\mathrm{O}^{\mathrm{iv}}$ & $2.803(4)$ & $\mathrm{Cu} 1-\mathrm{O} 5$ & $2.382(4)$ \\
\hline $\mathrm{Ba} 1-\mathrm{O}^{\mathrm{i}}$ & $2.850(4)$ & $\mathrm{Cu} 1-\mathrm{Ba}^{\mathrm{vii}}$ & $3.6787(10)$ \\
\hline $\mathrm{Ba} 1-\mathrm{O}^{\mathrm{iv}}$ & $2.904(4)$ & $\mathrm{Cu} 1-\mathrm{Ba} 1^{\text {viii }}$ & 3.9494 (12) \\
\hline $\mathrm{Ba} 1-\mathrm{O} 1^{\mathrm{ii}}$ & $2.938(4)$ & $\mathrm{O} 1-\mathrm{As} 1^{\mathrm{vii}}$ & $1.685(4)$ \\
\hline $\mathrm{Ba} 1-\mathrm{O}^{\mathrm{ii}}$ & $3.008(4)$ & $\mathrm{O} 1-\mathrm{Ba} 1^{\mathrm{viii}}$ & $2.803(4)$ \\
\hline $\mathrm{Ba} 1-\mathrm{As} 1$ & $3.5818(10)$ & $\mathrm{O} 1-\mathrm{Ba}^{\mathrm{vii}}$ & $2.938(4)$ \\
\hline $\mathrm{Ba} 1-\mathrm{Cu} 1^{\mathrm{ii}}$ & $3.6787(10)$ & $\mathrm{O} 2-\mathrm{As} 1^{\mathrm{vii}}$ & $1.654(4)$ \\
\hline $\mathrm{Ba} 1-\mathrm{As} 2$ & $3.7950(13)$ & $\mathrm{O} 2-\mathrm{Ba} 1^{\mathrm{ix}}$ & $2.683(5)$ \\
\hline $\mathrm{As} 1-\mathrm{O} 2^{\mathrm{ii}}$ & $1.654(4)$ & $\mathrm{O} 3-\mathrm{As} 1^{\mathrm{ix}}$ & $1.663(4)$ \\
\hline $\mathrm{As} 1-\mathrm{O} 3^{\mathrm{i}}$ & $1.663(4)$ & $\mathrm{O} 3-\mathrm{Ba} 1^{\mathrm{ix}}$ & $2.850(4)$ \\
\hline As1-O $1^{\mathrm{ii}}$ & $1.685(4)$ & $\mathrm{O} 4-\mathrm{As} 2^{\mathrm{ix}}$ & $1.752(4)$ \\
\hline As $1-\mathrm{O} 4$ & $1.744(4)$ & $\mathrm{O} 5-\mathrm{As} 2^{\mathrm{vi}}$ & $1.650(4)$ \\
\hline As $1-B a 1^{\mathrm{ii}}$ & $3.8019(11)$ & $\mathrm{O} 5-\mathrm{Ba} 1^{\text {viii }}$ & $2.904(4)$ \\
\hline $\mathrm{As} 1-\mathrm{Ba}^{\mathrm{v}}$ & $3.8295(9)$ & $\mathrm{O} 5-\mathrm{Ba} 1^{\mathrm{vii}}$ & $3.008(4)$ \\
\hline As $2-O 5^{\mathrm{vi}}$ & $1.650(4)$ & $\mathrm{O} 6-\mathrm{Ba}^{\mathrm{vii}}$ & $2.764(4)$ \\
\hline As2-O6 & $1.685(4)$ & $\mathrm{O} 7-\mathrm{As} 2^{\mathrm{iii}}$ & $1.698(4)$ \\
\hline As $2-O 7^{\mathrm{iii}}$ & $1.698(4)$ & $\mathrm{O} 7-\mathrm{Ba} 1^{\mathrm{iii}}$ & $2.803(4)$ \\
\hline $\mathrm{As} 2-\mathrm{O} 4^{\mathrm{i}}$ & $1.752(4)$ & & \\
\hline $\mathrm{O} 2-\mathrm{Ba} 1-\mathrm{O} 2^{\mathrm{i}}$ & $153.81(10)$ & $\mathrm{O} 22^{\mathrm{ii}}-\mathrm{As} 1-\mathrm{Ba} 1$ & $131.49(17)$ \\
\hline $\mathrm{O} 2-\mathrm{Ba} 1-\mathrm{O} 6^{\mathrm{ii}}$ & $125.38(13)$ & $\mathrm{O} 3^{\mathrm{i}}-\mathrm{As} 1-\mathrm{Ba} 1$ & $51.16(15)$ \\
\hline $\mathrm{O} 2^{\mathrm{i}}-\mathrm{Ba} 1-\mathrm{O} 6^{\mathrm{ii}}$ & $76.68(13)$ & $\mathrm{O}^{\mathrm{ii}}-\mathrm{As} 1-\mathrm{Ba} 1$ & $54.33(15)$ \\
\hline $\mathrm{O} 2-\mathrm{Ba} 1-\mathrm{O} 7^{\mathrm{iii}}$ & $104.89(14)$ & $\mathrm{O} 4-\mathrm{As} 1-\mathrm{Ba} 1$ & $120.01(15)$ \\
\hline $\mathrm{O} 2^{\mathrm{i}}-\mathrm{Ba} 1-\mathrm{O} 7^{\mathrm{iii}}$ & $91.88(14)$ & $\mathrm{O} 2^{\mathrm{ii}}-\mathrm{As} 1-\mathrm{Ba}^{\mathrm{ii}}$ & $35.13(16)$ \\
\hline $\mathrm{O} 6^{\mathrm{ii}}-\mathrm{Ba} 1-\mathrm{O} 7^{\mathrm{iii}}$ & $80.15(13)$ & $\mathrm{O} 3^{\mathrm{i}}-\mathrm{As} 1-\mathrm{Ba}^{\mathrm{ii}}$ & $82.79(16)$ \\
\hline $\mathrm{O} 2-\mathrm{Ba} 1-\mathrm{O}^{\mathrm{iv}}$ & $90.97(13)$ & $\mathrm{O} 1^{\mathrm{ii}}-\mathrm{As} 1-\mathrm{Ba}^{\mathrm{ii}}$ & $113.44(15)$ \\
\hline $\mathrm{O} 2^{\mathrm{i}}-\mathrm{Ba} 1-\mathrm{O} 1^{\mathrm{iv}}$ & $71.47(13)$ & $\mathrm{O} 4-\mathrm{As} 1-\mathrm{Ba} 1^{\mathrm{ii}}$ & $133.13(14)$ \\
\hline $\mathrm{O} 6^{\mathrm{ii}}-\mathrm{Ba} 1-\mathrm{O} 1^{\mathrm{iv}}$ & $142.42(13)$ & $\mathrm{Ba} 1-\mathrm{As} 1-\mathrm{Ba}^{\mathrm{ii}}$ & $101.86(2)$ \\
\hline $\mathrm{O} 7^{\mathrm{iii}}-\mathrm{Ba} 1-\mathrm{O} 1^{\mathrm{iv}}$ & $81.45(12)$ & 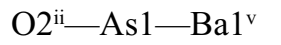 & $36.33(16)$ \\
\hline $\mathrm{O} 2-\mathrm{Ba} 1-\mathrm{O} 3^{\mathrm{i}}$ & $96.66(13)$ & $\mathrm{O} 3^{\mathrm{i}}-\mathrm{As} 1-\mathrm{Ba}^{\mathrm{v}}$ & $152.13(16)$ \\
\hline $\mathrm{O} 2^{\mathrm{i}}-\mathrm{Ba} 1-\mathrm{O} 3^{\mathrm{i}}$ & $76.64(13)$ & $\mathrm{O} 1^{\mathrm{ii}}-\mathrm{As} 1-\mathrm{Ba}^{\mathrm{v}}$ & $89.59(15)$ \\
\hline $\mathrm{O} 6^{\mathrm{ii}}-\mathrm{Ba} 1-\mathrm{O} 3^{\mathrm{i}}$ & $70.14(13)$ & $\mathrm{O} 4-\mathrm{As} 1-\mathrm{Ba}^{\mathrm{v}}$ & $90.19(15)$ \\
\hline $\mathrm{O} 7^{\mathrm{iii}}-\mathrm{Ba} 1-\mathrm{O} 3^{\mathrm{i}}$ & $149.83(13)$ & $\mathrm{Ba} 1-\mathrm{As} 1-\mathrm{Ba}^{\mathrm{v}}$ & 137.295 (19) \\
\hline $\mathrm{O} 1^{\mathrm{iv}}-\mathrm{Ba} 1-\mathrm{O} 3^{\mathrm{i}}$ & $119.53(12)$ & $\mathrm{Ba} 1^{\mathrm{ii}}-\mathrm{As} 1-\mathrm{Ba} 1^{\mathrm{v}}$ & 69.617 (19) \\
\hline $\mathrm{O} 2-\mathrm{Ba} 1-\mathrm{O}^{\mathrm{iv}}$ & $83.98(14)$ & $\mathrm{O} 5^{\mathrm{vi}}-\mathrm{As} 2-\mathrm{O} 6$ & $115.4(2)$ \\
\hline
\end{tabular}




$$
\begin{aligned}
& \mathrm{O} 2^{\mathrm{i}}-\mathrm{Ba} 1-\mathrm{O}^{\mathrm{iv}} \\
& \mathrm{O} 6^{\mathrm{ii}}-\mathrm{Ba} 1-\mathrm{O} 5^{\mathrm{iv}} \\
& \mathrm{O} 7^{\mathrm{iii}}-\mathrm{Ba} 1-\mathrm{O} 5^{\mathrm{iv}} \\
& \mathrm{O} 1^{\text {iv }}-\mathrm{Ba} 1-\mathrm{O}^{\text {iv }} \\
& \text { O3 }{ }^{\mathrm{i}}-\mathrm{Ba} 1-\mathrm{O}^{\mathrm{iv}} \\
& \mathrm{O} 2-\mathrm{Ba} 1-\mathrm{O}^{\mathrm{ii}} \\
& \mathrm{O} 2 \mathrm{i}-\mathrm{Ba} 1-\mathrm{O}^{\mathrm{ii}} \\
& \mathrm{O} 6 \text { ii }-\mathrm{Ba} 1-\mathrm{O} 1^{\mathrm{ii}}
\end{aligned}
$$

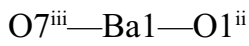

$$
\begin{aligned}
& \mathrm{O} 1^{\mathrm{iv}}-\mathrm{Ba} 1-\mathrm{O} 1^{\mathrm{ii}} \\
& \mathrm{O} 3^{\mathrm{i}}-\mathrm{Ba} 1-\mathrm{O}^{\mathrm{ii}} \\
& \mathrm{O} 5^{\mathrm{iv}}-\mathrm{Ba} 1-\mathrm{O} 1^{\mathrm{ii}} \\
& \mathrm{O} 2-\mathrm{Ba} 1-\mathrm{O}^{\mathrm{ii}} \\
& \mathrm{O} 2^{\mathrm{i}}-\mathrm{Ba} 1-\mathrm{O}^{\mathrm{ii}} \\
& \mathrm{O} 6^{\mathrm{ii}}-\mathrm{Ba} 1-\mathrm{O} 5^{\mathrm{ii}} \\
& \mathrm{O} 7^{\mathrm{iii}}-\mathrm{Ba} 1-\mathrm{O} 5^{\mathrm{ii}} \\
& \mathrm{O} 1^{\mathrm{iv}}-\mathrm{Ba} 1-\mathrm{O} 5^{\mathrm{ii}} \\
& \mathrm{O} 3^{\mathrm{i}}-\mathrm{Ba} 1-\mathrm{O}^{5^{\mathrm{ii}}} \\
& \mathrm{O} 5^{\mathrm{iv}}-\mathrm{Ba} 1-\mathrm{O} 5^{\mathrm{ii}} \\
& \mathrm{O} 1^{\mathrm{ii}}-\mathrm{Ba} 1-\mathrm{O} 5^{\mathrm{ii}} \\
& \text { O2-Ba1-As1 } \\
& \mathrm{O} 2 \mathrm{i}-\mathrm{Ba} 1-\mathrm{As} 1 \\
& \text { O6 }{ }^{\mathrm{ii}}-\mathrm{Ba} 1-\mathrm{As} 1 \\
& \text { O7 } 7^{\text {iii-Ba1-As1 }} \\
& \mathrm{O} 1^{\text {iv }} \text { - Ba1-As1 } \\
& \text { O3 }{ }^{\mathrm{i}} \text {-Ba1-As1 } \\
& \text { O5 }{ }^{\text {iv }-B a 1-A s 1 ~} \\
& \text { O1ii-Ba1-As1 } \\
& \text { O5 }{ }^{\mathrm{ii}} \text {-Ba1-As1 } \\
& \mathrm{O} 2-\mathrm{Ba} 1-\mathrm{Cu} 1^{\mathrm{ii}} \\
& \mathrm{O} 2^{\mathrm{i}}-\mathrm{Ba} 1-\mathrm{Cu}^{1 \mathrm{ii}} \\
& \text { O6 }{ }^{\mathrm{ii}}-\mathrm{Ba} 1-\mathrm{Cu} 1^{\mathrm{ii}} \\
& \mathrm{O} 7^{\mathrm{iii}}-\mathrm{Ba} 1-\mathrm{Cu} 1^{\mathrm{ii}} \\
& \mathrm{O} 1^{\text {iv }}-\mathrm{Ba} 1-\mathrm{Cu} 1^{\text {ii }} \\
& \mathrm{O} 3^{\mathrm{i}}-\mathrm{Ba} 1-\mathrm{Cu} 1^{\mathrm{ii}} \\
& \mathrm{O} 5^{\mathrm{iv}}-\mathrm{Ba} 1-\mathrm{Cu} 1^{\mathrm{ii}} \\
& \mathrm{O} 1^{\mathrm{ii}}-\mathrm{Ba} 1-\mathrm{Cu} 1^{\mathrm{ii}} \\
& \mathrm{O} 5^{\mathrm{ii}}-\mathrm{Ba} 1-\mathrm{Cu} 1^{\mathrm{ii}} \\
& \mathrm{As} 1-\mathrm{Ba} 1-\mathrm{Cu} 1^{\mathrm{ii}} \\
& \mathrm{O} 2-\mathrm{Ba} 1-\mathrm{As} 2 \\
& \mathrm{O} 2 \mathrm{i}-\mathrm{Ba} 1-\mathrm{As} 2 \\
& \text { O6 } 6 \text { ii-Ba1-As2 }
\end{aligned}
$$

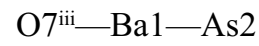

$$
\begin{aligned}
& \mathrm{O} 1^{\mathrm{iv}} \text { - Ba1-As2 } \\
& \text { O3 - Ba1-As2 } \\
& \mathrm{O} 5^{\mathrm{iv}} \text { - Ba1-As2 } \\
& \mathrm{O} 1^{\mathrm{ii}} \text {-Ba1-As2 } \\
& \text { O5 }{ }^{\mathrm{ii}} \text {-Ba1-As2 }
\end{aligned}
$$

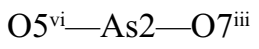

$$
\begin{aligned}
& \text { O6-As2-O } 7^{\text {iii }} \\
& \text { O5 }{ }^{\mathrm{vi}}-\mathrm{As} 2-\mathrm{O} 4^{\mathrm{i}} \\
& \mathrm{O} 6-\mathrm{As} 2-\mathrm{O} 4^{\mathrm{i}}
\end{aligned}
$$

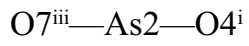

$$
\begin{aligned}
& \text { O5 }{ }^{\mathrm{vi}}-\mathrm{As} 2-\mathrm{Ba} 1 \\
& \text { O6-As2-Ba1 } \\
& \text { O7ii_-As2-Ba1 } \\
& \mathrm{O} 4 \mathrm{i}-\mathrm{As} 2-\mathrm{Ba} 1 \\
& \mathrm{O} 3-\mathrm{Cu} 1-\mathrm{O} 7 \\
& \mathrm{O} 3-\mathrm{Cu} 1-\mathrm{O} 6 \\
& \text { O7- } \mathrm{Cu} 1-\mathrm{O} 6 \\
& \mathrm{O} 3-\mathrm{Cu} 1-\mathrm{O} 1 \\
& \text { O7- } \mathrm{Cu} 1-\mathrm{O} 1 \\
& \text { O6- } \mathrm{Cu} 1-\mathrm{O} 1 \\
& \mathrm{O} 3-\mathrm{Cu} 1-\mathrm{O} 5 \\
& \mathrm{O} 7-\mathrm{Cu} 1-\mathrm{O} 5 \\
& \mathrm{O} 6-\mathrm{Cu} 1-\mathrm{O} 5 \\
& \mathrm{O} 1-\mathrm{Cu} 1-\mathrm{O} 5 \\
& \text { O3- } \mathrm{Cu} 1-\mathrm{Ba}^{\mathrm{vii}} \\
& \mathrm{O} 7-\mathrm{Cu} 1-\mathrm{Ba}^{\mathrm{vii}} \\
& \mathrm{O} 6-\mathrm{Cu} 1-\mathrm{Ba} 1^{\text {vii }} \\
& \mathrm{O} 1-\mathrm{Cu} 1-\mathrm{Ba} 1^{\text {vii }} \\
& \text { O5- } \mathrm{Cu} 1-\mathrm{Ba}^{\mathrm{vii}} \\
& \mathrm{O} 3-\mathrm{Cu} 1-\mathrm{Ba}^{\text {viii }} \\
& \mathrm{O} 7-\mathrm{Cu} 1-\mathrm{Ba}^{\text {viii }} \\
& \mathrm{O} 6-\mathrm{Cu} 1-\mathrm{Ba} 1^{\text {viii }} \\
& \mathrm{O} 1-\mathrm{Cu} 1-\mathrm{Ba}^{\text {viii }} \\
& \mathrm{O} 5-\mathrm{Cu} 1-\mathrm{Ba} 1^{\text {viii }} \\
& \mathrm{Ba} 1^{\mathrm{vii}}-\mathrm{Cu} 1-\mathrm{Ba}^{\mathrm{viii}} \\
& \text { As1 }{ }^{\mathrm{vii}}-\mathrm{O} 1-\mathrm{Cu} 1 \\
& \text { As1 } 1^{\text {vii }-O} 1-B a 1^{\text {viii }} \\
& \mathrm{Cu} 1-\mathrm{O} 1-\mathrm{Ba} 1^{\text {viii }} \\
& \text { As } 1^{\text {vii }}-\mathrm{O} 1-\mathrm{Ba} 1^{\mathrm{vii}} \\
& \mathrm{Cu} 1-\mathrm{O} 1-\mathrm{Ba}^{\mathrm{vii}} \\
& \text { Ba } 1^{\text {viii-O } 1-B a 1^{\text {vii }}} \\
& \text { As1 } 1 \text { vii-O2-Ba1 } \\
& \text { As1 }{ }^{\text {vii-O2- } 2-B a 1^{\text {ix }}} \\
& \mathrm{Ba} 1-\mathrm{O} 2-\mathrm{Ba}^{1 \mathrm{x}} \\
& \mathrm{As} 11^{\mathrm{ix}}-\mathrm{O} 3-\mathrm{Cu} 1 \\
& \mathrm{As} 1^{\mathrm{ix}}-\mathrm{O} 3-\mathrm{Ba}^{1 \mathrm{ix}} \\
& \mathrm{Cu} 1-\mathrm{O} 3-\mathrm{Ba} 1^{\mathrm{ix}} \\
& \text { As1-O4-As2 }{ }^{\text {ix }} \\
& \text { As2 }{ }^{\mathrm{vi}}-\mathrm{O} 5-\mathrm{Cu} 1 \\
& \text { As } 2^{\text {vi }}-\mathrm{O} 5-\mathrm{Ba} 1^{\text {viii }} \\
& \mathrm{Cu} 1-\mathrm{O} 5-\mathrm{Ba}^{\mathrm{viii}} \\
& \text { As2 }{ }^{\text {vi }}-\mathrm{O} 5-\mathrm{Ba} 1^{\mathrm{vii}} \\
& \mathrm{Cu} 1-\mathrm{O} 5-\mathrm{Ba}^{\mathrm{vii}}
\end{aligned}
$$

$117.7(2)$

$108.5(2)$

$104.0(2)$

$104.5(2)$

$105.4(2)$

$159.52(16)$

$74.00(15)$

42.97 (15)

$90.18(14)$

87.90 (19)

$158.73(19)$

$89.01(18)$

$91.48(18)$

$172.97(17)$

$89.03(18)$

$109.54(17)$

$113.23(17)$

$90.99(17)$

$73.56(16)$

$142.53(14)$

$128.98(13)$

$47.66(12)$

$52.83(12)$

54.59 (11)

75.98 (14)

$143.84(13)$

$117.12(13)$

42.25 (12)

46.97 (11)

$69.55(2)$

$112.7(2)$

$133.9(2)$

108.69 (17)

97.90 (18)

$93.86(15)$

98.69 (13)

$123.6(2)$

$122.2(2)$

110.24 (15)

138.7 (3)

$101.80(18)$

114.94 (18)

125.3 (2)

122.7 (2)

126.4 (2)

96.18 (14)

121.3 (2)

85.23 (12) 


$\begin{array}{llll}\text { As1-Ba1-As2 } & 154.659(18) & \mathrm{Ba}^{\mathrm{viii}}-\mathrm{O} 5-\mathrm{Ba} 1^{\mathrm{vii}} & 94.90(13) \\ \mathrm{Cu} 1^{\mathrm{ii}}-\mathrm{Ba} 1-\mathrm{As} 2 & 105.81(2) & \mathrm{As} 2-\mathrm{O} 6-\mathrm{Cu} 1 & 120.0(2) \\ \mathrm{O}^{\mathrm{ii}}-\mathrm{As} 1-\mathrm{O} 3^{\mathrm{i}} & 115.9(2) & \mathrm{As} 2-\mathrm{O} 6-\mathrm{Ba} 1^{\mathrm{vii}} & 132.3(2) \\ \mathrm{O} 2^{\mathrm{ii}}-\mathrm{As} 1-\mathrm{O} 1^{\mathrm{ii}} & 112.0(2) & \mathrm{Cu} 1-\mathrm{O} 6-\mathrm{Ba} 1^{\mathrm{vii}} & 100.32(17) \\ \mathrm{O} 3^{\mathrm{i}}-\mathrm{As} 1-\mathrm{O} 1^{\mathrm{ii}} & 105.5(2) & \mathrm{As} 2^{\mathrm{iii}}-\mathrm{O} 7-\mathrm{Cu} 1 & 116.1(2) \\ \mathrm{O} 2^{\mathrm{ii}}-\mathrm{As} 1-\mathrm{O} 4 & 108.5(2) & \mathrm{As} 2^{\mathrm{iii}}-\mathrm{O} 7-\mathrm{Ba} 1^{\mathrm{iii}} & 112.6(2) \\ \mathrm{O} 3^{\mathrm{i}}-\mathrm{As} 1-\mathrm{O} 4 & 106.6(2) & \mathrm{Cu} 1-\mathrm{O} 7-\mathrm{Ba} 1^{\mathrm{iii}} & 130.72(19) \\ \mathrm{O} 1^{\mathrm{ii}}-\mathrm{As} 1-\mathrm{O} 4 & 108.0(2) & & \end{array}$

Symmetry codes: (i) $-x+1 / 2, y+1 / 2,-z+1 / 2$; (ii) $x-1, y, z$; (iii) $-x+1,-y+1,-z$; (iv) $-x+3 / 2, y+1 / 2,-z+1 / 2$; (v) $-x-1 / 2, y-1 / 2,-z+1 / 2$; (vi) $-x+2,-y+1,-z$; (vii) $x+1, y, z$; (viii) $-x+3 / 2, y-1 / 2,-z+1 / 2$; (ix) $-x+1 / 2, y-1 / 2,-z+1 / 2$. 